\title{
Dynamical Updating Fuzzy Rough Approximations for Hybrid Data under the Variation of Attribute Values
}

\author{
Anping Zeng ${ }^{\mathrm{a}, \mathrm{b}}$, Tianrui $\mathrm{Li}^{\mathrm{a}, *}$, Jie $\mathrm{Hu}^{\mathrm{a}}$, Hongmei Chen ${ }^{\mathrm{a}}$, Chuan $\mathrm{Luo}^{\mathrm{c}}$ \\ ${ }^{a}$ School of Information Science and Technology, Southwest Jiaotong University, Chengdu 611756, China \\ ${ }^{b}$ School of Computer and Information Engineering, Yibin University, Yibin 644007, China \\ ${ }^{c}$ College of Computer Science, Sichuan University, Chengdu 610065, China
}

\begin{abstract}
With the development of the Internet of Things (IoT), more and more hybrid data is being collected by information systems, which are known as Hybrid Information Systems (HIS). Based on a new hybrid distance, novel Gaussian kernel Fuzzy Rough Sets (FRS) for HIS were constructed in our previous study. In real-world applications, with the deepening of cognition and improvements in technology, attribute values in an information system often evolve over time; in particular, there are three cases: when missing values are imputed, error values are corrected, and the values are coarsened or refined. This has posed challenges to developing efficient data analysis algorithms. In this paper, the changing mechanisms of the attribute values and fuzzy equivalence relations in FRS are analyzed. FRS approaches for incrementally updating approximations in HIS are presented. Moreover, two corresponding incremental algorithms are developed. Finally, extensive experiments on eight data sets from the University of California, Irvine (UCI) and an artificial data set show that incremental approaches can effectively improve the performance of updating approximations and not only significantly shorten the computational time, but also increase approximation classification accuracies.
\end{abstract}

Keywords: Fuzzy rough sets; Incremental learning; Hybrid information systems; Big data.

\section{Introduction}

Current explosive growth and continued access to various data has formed more and more big data systems. Big data has the characteristics of the " $5 \mathrm{Vs}$ ", i.e., volume, variety, velocity, veracity, and value [11]. Volume is the quantity of samples in the data set and is very large. Variety implies that the data exists with hybrid formats or comes from multiple sources; more specifically, hybrid data may consist of Boolean, categorical, real-valued, set-valued, intervalvalued, missing value, text, image, video, audio, and sensor signal data. Velocity means that the data is updated drastically, i.e., the object set, attribute set, and attribute values may be changed dynamically. Veracity refers to the authenticity or genuineness of the data, and value means that a high yield will be achieved. Faced with the challenges of big data's $5 \mathrm{~V}$ characteristics, efficient data analysis algorithms that can improve the performance of knowledge discovery are urgently needed.

Rough Set Theory (RST), which captures the idea of indiscernibility among members of a set and utilizes a discrete formalism of set partitions, is an important mathematical tool in knowledge discovery [27, 36]. Information granularity is viewed as a crucial design asset that helps endow existing modeling paradigms and modeling practices with new conceptual and algorithmic features, thus making the resulting models more reflective of the complexities of real-world phenomena [37]. There are two approaches to granulate hyb,rid data. One approach is to composite

\footnotetext{
* Corresponding author.

Email addresses: zengap@126. com (Anping Zeng), trli@swjtu.edu.cn (Tianrui Li), jiehu@swjtu.edu.cn (Jie Hu), hmchen@swjtu.edu.cn (Hongmei Chen), cluo@scu.edu.cn (Chuan Luo)
} 
the variety of binary relations to granulate the hybrid data. The other approach is to design a new binary relation to granulate the hybrid data. RST can only utilize a binary relation to directly granulate categorical data. In order to granulate other types of data, many extending binary relations (i.e., the fuzzy relation [12], neighborhood relation [28], tolerance relation [39], dominance relation [13], etc.) are presented.

When granulating hybrid data, many scholars composite a variety of binary relations. In order to generate a decision rule set in hybrid data, which includes categorical, real-valued data, An et al. composited three relations, in particular, indiscernibility, similarity, and the dominance relations and constructed corresponding rough approximations [1]. Zhang et al. developed a composite rough set with the goal of dealing with composite information systems, which contain real-valued, set-valued, and interval-valued data [48, 49]. Based on composite rough sets, Chen et al. presented a probabilistic composite rough set [5]. Li et al. proposed a new composite rough set model under the tolerance and dominance relations [23]; furthermore, several strategies of decomposition and composition of granules in Dominance-based Rough Sets Approach (DRSA) were proposed for computing approximations in parallel [24]. Based on set-valued and ordered relations, Luo et al. proposed dynamic maintenance of approximations in set-valued ordered decision systems under the attribute of generalization [34].

Along with the increase of data types in composite information systems, the complexity of the granulating process will also increase, and the efficiency of knowledge discovery will decrease. Therefore, many efforts have been devoted to another alternative approach to granulate hybrid data. Hu et al. presented neighborhood relation-based information granulation for hybrid data containing categorical and real-valued data [14]. To deal with hybrid information systems, which contain numerical and incomplete attributes, Zhao et al. proposed a generalized incomplete rough set model based on the neighborhood relation [51]. Furthermore, Hu et al. utilized the fuzzy equivalence relation to granulate categorical and real-valued data based on a novel Fuzzy Rough Set (FRS) [17]. In this model, the value difference is one between two different categorical values; otherwise, the value difference is considered to be zero. In [40], Sun et al. further presented the interval-valued FRS model based on interval-valued fuzzy information systems using a binary interval-valued fuzzy relation. Pedrycz et al. elaborated on distributed fuzzy modeling and ensuing granular fuzzy modeling and designed a hierarchical approach to fuzzy modeling [38]. Hu et al. developed a fuzzy rule-based model with interactive rules and granular generalization [18].

In combination with the Euclidean distance, Hu et al. first introduced Gaussian kernels to acquire fuzzy relations between samples, which are described by fuzzy or real-valued attributes, in order to generate fuzzy information granules in the approximation space [15, 16]. Based on the Euclidean distance, we presented an Hybrid Distance (HD), which can deal with hybrid data consisting of Boolean, categorical, real-valued, and set-valued data, and proposed a novel Gaussian kernel FRS [44]. It is infeasible to consider interval-valued and unstructured data using the Euclidean distance and HD distance directly. Furthermore, we introduce an extended hybrid distance and a new FRS model.

Today, different types of data collected from different applications increase rapidly, and the data may evolve over times, i.e., the objects, attributes, and attribute values may change dynamically. Though we can discover the knowledge in Hybrid Information Systems (HIS) using the static FRS model, it is very time-consuming because of the complexity brought about by the hybrid attributes and dynamical characteristics. Therefore, knowledge discovery becomes more inefficient. Incremental learning is essential for solving these problems. It is feasible when the data structure and information of the previous data are stored and the old data does not need to be rescanned. Then, the relationships between the added new data and the stored data structure can be established in order to obtain new knowledge. Therefore, incremental learning can improve the efficiency of knowledge discovery in HIS. Under Rough Fuzzy Sets (RFS) and other extended RST models, some incremental learning approaches in dynamic information systems have been presented.

Variation of objects has been widely considered in incremental learning. Chen et al. developed an incremental updating approximations method under Variable Precision Rough Sets (VPRS) [7]. Liu et al. proposed an incremental approach for inducing interesting knowledge based on both accuracy and coverage [29]. Wang et al. designed a matrix-based method for incremental updating approximations [41]. Zhang et al. proposed a Neighborhood Rough Sets (NRS) approach for dynamic maintenance of approximations [47]. Li et al. presented a dynamic maintenance of approximations in DRSA [20]. Furthermore, Luo et al. presented an incremental approach for updating approximations in set-valued ordered information systems [33]. Zhang et al. constructed a composite rough set model for dynamic data mining [48].

In the case of the variation of an attribute set, Chan presented an incremental method for updating the approximations of Pawlak's RST [3]. Li et al. proposed an incremental approach for updating the approximations and further 
extended it to the framework of similar relations and characteristic relations based on RST [25, 26]. In Rough Fuzzy Sets (RFS), Cheng developed two incremental approaches to quickly update approximations by updating the boundary region and cut sets [9]. Furthermore, Zhang et al. discussed the strategies and propositions for incrementally updating approximations under VPRS [46]. Li et al. introduced a kind of dominance matrix to calculate P-dominating sets and P-dominated sets in DRSA and proposed incremental algorithms for updating approximations of DRSA [22]. In set-valued ordered decision systems, Luo et al. focused on maintaining approximations dynamically under attribute generalization [34]. Zeng et al. proposed an FRS approach for updating approximations and then applied them to feature selection [44]. Liu et al. presented a probability rough set approach for incrementally updating approximations [29]. When objects and attributes are added simultaneously under the framework of Decision-Theoretic Rough Set (DTRS), Chen et al. proposed a DTRS approach for dynamic data mining [6].

In real-world applications, dynamic changing attribute values are very common. For example, missing values are inserted, error values are corrected, and values are coarsened or refined. Chen et al. proposed concepts related to coarsening and refining of attribute values and presented some incremental approaches for updating approximations in Pawlak's RST [8]. Liu et al. illustrated an incremental approach to obtain interesting knowledge [30]. Li et al. proposed incremental algorithms for updating approximations of DRSA [21]. Luo et al. developed dynamic rough set approaches in set-valued decision systems [32, 35]. Zeng et al. presented an incremental approach for updating approximations in RFS [43]. In this paper, we discuss FRS approaches for incremental updating approximations, which can be used in many domains of Internet of Things (IoT) and big data such as medical IoT, industrial safety monitoring, smart city, and environmental monitoring.

The remainder of this paper is organized as follows. In Section 2, preliminaries are introduced. In Section 3, the generating methods of fuzzy information granules in HIS are presented. In Section 4, the non-incremental updating algorithm for updating approximations is presented. In Section 5, the incremental updating principles and algorithms for updating approximations are analyzed under the variation of attribute values. In Section 6, some experiments are designed to evaluate the performance of the proposed incremental algorithms. Finally, in Section 7, we conclude our paper.

\section{Preliminaries}

In this section, we briefly introduce basic concepts of rough sets and FRS $[12,36]$.

\subsection{Pawlak's rough sets}

Definition 1. [36] An information system is defined as a pair $\langle U, A\rangle$, where $U$ is a non-empty finite set of objects, $A=C \cup D$ is a non-empty finite set of attributes, $C$ denotes the set of condition attributes, $D$ denotes the set of decision attributes, and $C \cap D=\emptyset$. Each attribute $a \in A$ is associated with a set $V_{a}$ of its value called the domain of a.

The RST describes a crisp subset of a universe by two definable subsets called lower and upper approximations. By using the lower and upper approximations, the knowledge hidden in information systems can be discovered and expressed in the form of decision rules.

Definition 2. [36] Let $U$ be a finite universe with $U \neq \emptyset$. $R$ is an equivalence relation on $U$ if $\forall x, y, z \in U$, we have:

(1) Reflexivity: $R(x, x)=1$,

(2) Symmetry: $R(x, y)=R(y, x)$,

(3) Transitivity: $R(x, y)=R(y, z) \Rightarrow R(x, y)=R(x, z)$.

The equivalence relation partitions the universe into a family of disjoint subsets called equivalence classes. The equivalence class including $x$ is denoted by $[x]_{R}$.

Definition 3. [36] Let $<U, R>$ be a Pawlak approximation space with universe $U \neq \emptyset . R \subseteq U \times U$ is an equivalence relation on $U$. $U / R$ denotes the family of all equivalence classes $R$, and $[x]_{R}$ denotes an equivalence class of $R$ containing an element $x \in U$. For any $X \subseteq U$, the lower and upper approximations of $X$ are defined, respectively, as follows:

$$
\left\{\begin{array}{l}
\underline{R} X=\left\{x \in U \mid[x]_{R} \subseteq X\right\}, \\
\bar{R} X=\left\{x \in U \mid[x]_{R} \cap X \neq \emptyset\right\} .
\end{array}\right.
$$




\subsection{Fuzzy rough sets}

Definition 4. [4] Let $U$ be a non-empty, finite universe and $\tilde{A}$ be a fuzzy subset on $U . \forall u \in U, \tilde{A}$ is defined by the following real function:

$$
\begin{aligned}
\mu_{\tilde{A}}: \quad U & \rightarrow[0,1] \\
u & \mapsto \mu_{\tilde{A}}(u),
\end{aligned}
$$

where $\mu_{\tilde{A}}(u)$ (or $\tilde{A}(u)$, for short) is the membership degree of $\tilde{A}$.

Let $U=\left\{x_{1}, x_{2}, \ldots, x_{n}\right\}$. A fuzzy set $\tilde{A}$ can be represented by $\left\{\tilde{A}\left(x_{1}\right) / x_{1}, \tilde{A}\left(x_{2}\right) / x_{2}, \ldots, \tilde{A}\left(x_{n}\right) / x_{n}\right\}$.

Definition 5. [12] Let $U$ be a finite universe with $U \neq \emptyset$ and $R \in F(U \times U)$. $R$ is a fuzzy equivalence relation on $U$ if $\forall x, y, z \in U$, we have:

(1) Reflexivity: $R(x, x)=1$,

(2) Symmetry: $R(x, y)=R(y, x)$,

(3) Min-max transitivity: $\min (R(x, y), R(y, z)) \leq R(x, z)$.

A binary operator $T$ on the unit interval $I=[0,1]$ is said be a triangular norm if $\forall a, b, c \in I$, the following conditions are satisfied:

(1) Commutativity: $T(a, b)=T(b, a)$,

(2) Associativity: $T(T(a, b), c)=T(a, T(b, c))$,

(3) Monotonicity: $a \leq c, b \leq d \Rightarrow T(a, b) \leq T(c, d)$,

(4) Boundary condition: $T(a, 1)=a, T(1, a)=a$.

We say that a binary operator $S$ on the unit interval $I$ is a triangular-conorm ( $t$-conorm or $s$-norm, for short) if it is increasing, associative, and commutative and satisfies the boundary condition of the form $S(a, 0)=a, \forall a \in[0,1]$.

A negator $N$ is a decreasing mapping from $[0,1] \rightarrow[0,1]$ that satisfies $N(0)=1$ and $N(1)=0$. The negator $N_{s}(a)=1-a$ is usually referred to as the standard negator. A negator $N$ is said to be involutive if $N(N(a))=a$, $\forall a \in[0,1]$. If $S(a, b)=N(T(N(a), N(b)))$ and $T(a, b)=N(S(N(a), N(b))), \forall a, b \in[0,1]$, we say $T$ and $S$ are dual with respect to $N$.

It is clear that $T_{\cos }(a, b)=\max \left\{a b-\sqrt{1-a^{2}} \sqrt{1-b^{2}}, 0\right\}$ is a $T$-norm, and its dual $S_{c o s}(a, b)=\min \{a+b-a b+$ $\left.\sqrt{2 a-a^{2}} \sqrt{2 b-b^{2}}, 1\right\}$.

Definition 6. [12] Let $U$ be a finite universe with $U \neq \emptyset$ and $R \in F(U \times U)$. $R$ is a $T$-fuzzy equivalence relation on $U$ if $\forall x, y, z \in U$, we have:

(1) Reflexivity: $R(x, x)=1$,

(2) Symmetry: $R(x, y)=R(y, x)$,

(3) $T$-transitivity: $T(R(x, y), R(y, z)) \leq R(x, z)$.

Since $T_{\cos }$-norm is a $T$-norm, we have $T_{\cos }(R(x, y), R(y, z)) \leq R(x, z)$. Therefore, $R$ is a $T_{\cos }$-fuzzy equivalence relation on $U$.

Definition 7. [12] Let $R$ be a fuzzy equivalence relation on $U$ and $X$ be a fuzzy subset of $U$. The fuzzy lower and upper approximations of $X$ are defined, respectively, as follows:

$$
\left\{\begin{array}{l}
\underline{R} X(x)=\inf _{y \in U}\{\max (1-R(x, y), X(y))\}, \\
\bar{R} X(x)=\sup _{y \in U}\{\min (R(x, y), X(y))\} .
\end{array}\right.
$$

In [16], Hu et al. proposed a Gaussian Kernelized FRS model with $T_{\cos }$-norm $\left(T_{\cos }(a, b)=\max \left\{a b-\sqrt{1-a^{2}}\right.\right.$ $\left.\left.\sqrt{1-b^{2}}, 0\right\}\right)$, residual implication $\theta_{T_{c o s}}(a, b)=\left\{\begin{array}{ll}1, & a \leq b \\ a b+\sqrt{\left(1-a^{2}\right)\left(1-b^{2}\right)}, & a>b,\end{array}\right.$, and 


$$
\left\{\begin{array}{l}
R_{G \theta} \tilde{X}(x)=\inf _{y \in U} \theta_{T_{c o s}}\left(R_{G}(x, y), \tilde{X}(y)\right), \\
\overline{\overline{R_{G T}}} \tilde{X}(x)=\sup _{y \in U} T_{c o s}\left(R_{G}(x, y), \tilde{X}(y)\right),
\end{array}\right.
$$

where $R_{G}(x, y)=\exp \left(-\frac{\|x-y\|^{2}}{2 \delta^{2}}\right)$.

For a given HIS $=<U, C$ C $\cup D, V, f>, U / D=\left\{d_{i}\right\}, i=1,2, \ldots,|U / D|$. Let $d_{i}(x)=1, \forall x \in d_{i}$ and $d_{i}(x)=0, \forall x \notin d_{i}$. Equation (4) can be simplified as follows:

Proposition 1. [16] For $H I S=<U, C \cup D, V, f>, \forall d_{i} \in U / D$,

$$
\left\{\begin{array}{l}
\underline{R_{G}} d_{i}(x)=\inf _{y \notin d_{i}} \sqrt{1-R_{G}^{2}(x, y),} \\
\overline{R_{G}} d_{i}(x)=\sup _{y \in d_{i}} R_{G}(x, y) .
\end{array}\right.
$$

\section{Generation of fuzzy information granules in HIS}

Definition 8. An HIS can be denoted by $\left\langle U, C \cup D, V, f>\right.$, where $U$ is the set of objects, and $C=C^{r} \cup C^{s} \cup C^{c} \cup C^{b} \cup$ $C^{i} \cup C^{u}$, where $C^{r}$ is the real-valued attribute set, $C^{s}$ is the set-valued attribute set, $C^{c}$ is the categorical attribute set, $C^{b}$ is the boolean attribute set, $C^{i}$ is the interval attribute set, and $C^{u}$ is the unstructured attribute set. $D$ denotes the set of decision attributes. $C^{r}, C^{s}, C^{c}, C^{b}, C^{I}$, and $C^{u}$ are disjoint sets.

Example 1. Table 1 is an HIS with categorical attribute "Urinary Color", Boolean attribute "Urinary Tuberculosis", real-valued attribute " $P H$ ", set-valued attribute "Urinary Irritation", two interval-valued attributes " $R B C$ " and "WBC", an unstructured attribute (image attribute) " $C T$ "; these attributes are denoted by $a_{1}, a_{2}, a_{3}, a_{4}, a_{5}, a_{6}, a_{7}$, respectively. Finally, $d$ is a decision attribute. Here, "?" denotes an unknown value. Furthermore, "RBC" is short for Red Blood Cell, "WBC" is short for White Blood Cell, and "M", "U", and "P" denote Micturition, Urgent urination, and Pain, respectively.

\subsection{Hybrid distance}

In HIS, hybrid attributes may consist of Boolean, categorical, real-valued, set-valued, interval-valued, text, image, video, audio, and sensor signal data. In [44], we proposed an HD distance, which can deal with select hybrid data including Boolean, categorical, real-valued, and set-valued data. However, the HD distance cannot deal with other types of data such as interval-valued, text, image, video, audio, and sensor signals. Therefore, an updated HD distance (HD-I) for hybrid attributes is presented.

Definition 9. [44] Let $H I S=<U, C \cup D, V, f>. \forall x, y \in U, \forall a \in C^{c} \cup C^{b}$, $a(x)$ and $a(y)$ denote the values of $x$ and $y$ under a, respectively. The value difference of Boolean $(v d b)$ attributes is defined as follows:

$$
v d b(a(x), a(y))= \begin{cases}0, & a(x)=a(y), \\ 1, & a(x) \neq a(y) .\end{cases}
$$

Definition 10. [42] Let $H I S=<U, C \cup D, V, f>. \forall x, y \in U, \forall a \in C^{r}, a(x)$ and $a(y)$ denote two values of $x$ and $y$ under a, respectively. The value difference of real-valued attributes is defined as follows:

$$
v d r(a(x), a(y))=\frac{|a(x)-a(y)|}{4 \delta_{a}},
$$

where $\delta_{a}$ is the standard deviation under attribute a. 
Table 1: A hybrid information system

\begin{tabular}{|c|c|c|c|c|c|c|c|c|}
\hline$U$ & $\begin{array}{l}\text { Urinary } \\
\text { Color }\left(a_{1}\right)\end{array}$ & $\begin{array}{c}\text { Urinary } \\
\text { Tuberculosis }\left(a_{2}\right)\end{array}$ & $\begin{array}{l}\mathbf{P H} \\
\left(a_{3}\right)\end{array}$ & $\begin{array}{c}\text { Urinary } \\
\text { Irritation }\left(a_{4}\right)\end{array}$ & $\begin{array}{c}\mathbf{R B C} \\
\left(a_{5}\right)\end{array}$ & $\begin{array}{c}\text { WBC } \\
\left(a_{6}\right)\end{array}$ & $\begin{array}{l}\mathrm{CT} \\
\left(a_{7}\right)\end{array}$ & $d$ \\
\hline$x_{1}$ & Pink & - & 4.0 & $\{\mathrm{M}, \mathrm{U}, \mathrm{P}\}$ & {$[1,4]$} & {$[3,7]$} & & Nephritis \\
\hline$x_{2}$ & Pink & - & 4.5 & $\{\mathrm{M}, \mathrm{U}, \mathrm{P}\}$ & {$[2,4]$} & {$[2,6]$} & & Nephritis \\
\hline$x_{3}$ & Red & $?$ & 3.8 & $\{\mathrm{M}\}$ & {$[1,3]$} & {$[1,4]$} & & Nephritis \\
\hline$x_{4}$ & Pink & + & 3.6 & $\{\mathrm{U}\}$ & {$[2,5]$} & {$[5,7]$} & & hrotuberculosis \\
\hline$x_{5}$ & Red & + & 3.5 & $\{\mathrm{U}\}$ & {$[1,4]$} & {$[6,7]$} & & otuberculosis \\
\hline$x_{6}$ & Yellow & - & 5.5 & $\{\mathrm{U}, \mathrm{P}\}$ & {$[0,2]$} & {$[0,5]$} & & Health \\
\hline$x_{7}$ & Yellow & $?$ & $?$ & $\{\mathrm{P}\}$ & {$[0,2]$} & {$[1,4]$} & & Health \\
\hline$x_{8}$ & Yellow & - & 4.1 & $\{\mathrm{M}, \mathrm{U}, \mathrm{P}\}$ & {$[2,4]$} & {$[4,6]$} & & Nephritis \\
\hline$x_{9}$ & $?$ & - & 5.6 & $\{\mathrm{U}\}$ & {$[0,2]$} & {$[2,4]$} & & Health \\
\hline
\end{tabular}

Definition 11. [42] Let $H I S=<U, C \cup D, V, f>, \forall x, y \in U, \forall a \in C, a(x)$ and $a(y)$ denote two values of $x$ and $y$ under $a$, respectively. Here, "?" denotes an unknown value. If $a(x)=$ ? or $a(y)=$ ? and $x \neq y$, the value difference of unknown values (vdu) is defined as follows:

$$
v d u(a(x), a(y))=1
$$

Definition 12. [44] Let $H I S=<U, C \cup D, V, f>. \forall x, y \in U$ and $\forall a \in C^{s}$, let $V_{a}$ be the domain of $a$. a $a(x)$ and $a(y)$ denote two values of $x$ and $y$ under a, respectively. The value difference of set-valued (vds) attributes is defined as follows:

$$
v d s(a(x), a(y))=\frac{|(a(x) \cup a(y))-(a(x) \cap a(y))|}{s},
$$

where $s$ is the maximum cardinal number in the subset of $V_{a}$.

Definition 13. [10] Let $A=\left[a^{-}, a^{+}\right]$and $B=\left[b^{-}, b^{+}\right]$be two interval values. The possible degree of $A$ relative to $B$ is

$$
P_{(A \geq B)}=\min \left\{1, \max \left\{\frac{a^{+}-b^{-}}{\left(a^{+}-a^{-}\right)+\left(b^{+}-b^{-}\right)}, 0\right\}\right\} .
$$

In [10], Dai et al. proposed the definition of the similarity measure between two interval values under the possible degree.

Definition 14. [10] Let $A=\left[a^{-}, a^{+}\right]$and $B=\left[b^{-}, b^{+}\right]$be two interval values. The similarity measure between $A$ and $B$ is defined by

$$
\operatorname{sim}(A, B)=1-\left|P_{(a(x) \geq a(y))}-P_{(a(y) \geq a(x))}\right|,
$$


where $P_{(A \geq B)}$ and $P_{(B \geq A)}$ are viewed as the possible degree of $A$ greater than $B$ and the possible degree of $B$ greater than $A$, respectively.

According to Definition 14, the distance measure between two interval values is defined as follows.

Definition 15. [10] Let $H I S=<U, C \cup D, V, f>, \forall x, y \in U$ and $\forall a \in C^{i}, a(x)$ and a(y) are two attribute values of $x$ and $y$ under a, respectively. The value difference of the interval-valued attribute is defined as follows:

$$
v \operatorname{di}(a(x), a(y))=\left|P_{(a(x) \geq a(y))}-P_{(a(y) \geq a(x))}\right|,
$$

where $P_{(A \geq B)}$ and $P_{(B \geq A)}$ are the possible degree of $A$ greater than $B$ and the possible degree of $B$ greater than $A$, respectively.

There are some other types of attributes (e.g., text, image, video, audio, and sensor signal) whose values are unstructured data in HIS. Through feature extraction, this data can be described as feature vectors. Using the Euclidean distance, we obtain the following definition.

Definition 16. Let $H I S=<U, C \cup D, V, f>. \forall x, y \in U$ and $\forall a \in C^{u}$, let $X$ and $Y$ denote two vector values of $x$ and $y$ under $a$, respectively. The value difference of unstructured attributes (vdus) is defined as follows:

$$
v d u s(X, Y)=\frac{\left\|X_{i}-Y_{i}\right\|}{\left\|V_{\max }-V_{\min }\right\|}
$$

where $\|$.$\| is the Euclidean distance and V_{\max }$ and $V_{\min }$ denote the maximum value and minimum value of $V_{a}$, respectively.

Example 2. In Table 1, $a_{7}\left(x_{1}\right)$ and $a_{7}\left(x_{3}\right)$ are two computed tomography $(C T)$ images. Using Gabor Wavelets (GWs), five-level and eight-direction decomposition is performed, and $40 \mathrm{GW}$ subbands are generated on both $a_{7}\left(x_{1}\right)$ and $a_{7}\left(x_{3}\right)$. The means and standard deviations of each subband are extracted, and all of the means and standard deviations are connected to an 80-dimensional feature vector [19]. The two vectors $a_{7}\left(x_{1}\right)$ and $a_{7}\left(x_{3}\right)$ are denoted by $X$ and $Y$, respectively. To be precise, $X=<4.55,8.12,10.01,15.43,25.46,6.44,9.75,11.03,8.69,12.99,8.91,10.24,9.85$, $7.53,8.79,11.11,12.59,12.71,11.69,10.57,10.75,13.38,15.08,16.86,24.89,9.32,10.15,12.07,12.40,11.37,7.44$, $10.61,9.21,8.34,10.01,5.26,7.36,9.49,8.94,10.47,20.29,46.73,34.14,62.14,74.99,39.67,47.56,61.04,31.09$, $46.33,42.08,51.66,22.13,16.09,17.38,116.12,77.23,66.81,55.07,53.70,102.18,144.39,117.80,71.05,58.04$, $100.70,118.01,103.11,52.51,46.89,46.81,70.78,43.85,25.03,18.45,26.62,43.92,41.93,43.29,34.90>$ and $Y=$ $<4.32,9.07,16.05,19.26,30.50,4.05,6.06,9.29,11.93,12.96,5.37,9.21,12.28,10.76,14.82,5.59,8.38,9.27,12.36$, $12.55,6.70,10.29,14.66,19.49,30.68,6.75,9.32,14.97,11.91,9.07,5.20,8.59,8.86,10.84,11.79,3.66,5.85,9.59$, $8.41,10.17,41.53,148.84,207.99,118.11,93.85,19.52,27.21,85.17,108.53,94.85,35.46,71.12,104.90,72.55$, $88.01,35.32,55.33,45.32,36.30,48.71,53.26,83.56,72.71,107.24,171.92,55.15,72.02,90.14,39.13,30.35$, $34.24,59.42,37.15,24.61,30.78,21.16,29.07,35.91,20.61,45.42>$. For the images, let $V_{\max }=<255, \ldots, 255>$ and $V_{\min }=<0, \ldots, 0>$; according to Definition 16, vdus $\left(a_{7}\left(x_{1}\right), a_{7}\left(x_{3}\right)\right)=\sqrt{\sum_{i=1}^{n}\left(X_{i}-Y_{i}\right)^{2}} / \sqrt{\sum_{i=1}^{n}(255-0)^{2}}=0.1432$.

In order to deal with hybrid and incomplete attributes and according to the above definitions, the HD-I distance can be defined as follows.

Definition 17. Given an HIS, the HD-I is defined by

$$
H D-I(x, y)=\sqrt{\sum_{a=1}^{m} d^{2}(a(x), a(y))},
$$


where $m$ is the number of attributes and

$$
d(a(x), a(y))= \begin{cases}1, & a(x)=? \text { or } a(y)=?, \\ v d b(a(x), a(y)), & a \text { is Boolean or categorical, } \\ v d s(a(x), a(y)), & \text { a is set-valued, } \\ v d r(a(x), a(y)), & \text { a is real-valued, } \\ v d i(a(x), a(y)), & \text { a is interval-valued, } \\ v d u s(a(x), a(y)), & \text { a is unstructured. }\end{cases}
$$

Example 3. Based on Example 1 and Definition 17, the following results hold:

(1) Because attribute $a_{1}$ is categorical, $d\left(a_{1}\left(x_{1}\right), a_{1}\left(x_{3}\right)\right)=v d b\left(a_{1}\left(x_{1}\right), a_{1}\left(x_{3}\right)\right)=1$.

(2) $a_{2}\left(x_{3}\right)=$ ?; therefore, $d\left(a_{2}\left(x_{1}\right), a_{2}\left(x_{3}\right)\right)=1$.

(3) Because attribute $a_{3}$ is real-valued, $d\left(a_{3}\left(x_{1}\right), a_{3}\left(x_{3}\right)\right)=\frac{a_{3}\left(x_{1}\right)-a_{3}\left(x_{3}\right)}{4 \delta_{a_{3}}}=\frac{4.0-3.8}{4 \times 3.0578}=0.02$.

(4) Because attribute $a_{4}$ is set-valued, $d\left(a_{4}\left(x_{1}\right), a_{4}\left(x_{3}\right)\right)=v d s\left(a_{4}\left(x_{1}\right), a_{4}\left(x_{3}\right)\right)=\frac{2}{3}=0.67$.

(5) Because $a_{5}$ is interval-valued, $d\left(a_{5}\left(x_{1}\right), a_{5}\left(x_{3}\right)\right)=v d i\left(a_{5}\left(x_{1}\right), a_{5}\left(x_{3}\right)\right)=\left|\frac{4-1}{(4-1)+(3-1)}-\frac{3-1}{(3-1)+(4-1)}\right|=0.2$.

(6) Because $a_{6}$ is interval-valued, $d\left(a_{6}\left(x_{1}\right), a_{6}\left(x_{3}\right)\right)=v$ di $\left(a_{6}\left(x_{1}\right), a_{6}\left(x_{3}\right)\right)=\left|\frac{7-1}{(7-3)+(4-1)}-\frac{4-3}{(4-1)+(7-3)}\right|=0.7142$.

(7) Because $a_{7}$ is unstructured-valued, $d\left(a_{7}\left(x_{1}\right), a_{7}\left(x_{3}\right)\right)=v d u s\left(a_{7}\left(x_{1}\right), a_{7}\left(x_{3}\right)\right)=0.1432$.

Therefore,

$H D-I\left(x_{1}, x_{3}\right)=\left(\sum_{a=1}^{7} d^{2}(a(x), a(y))\right)^{1 / 2}=\sqrt{1^{2}+1^{2}+0.02^{2}+0.67^{2}+0.2^{2}+0.7142^{2}+0.1432^{2}}=1.74$.

\subsection{Generation of fuzzy information granules with hybrid attributes}

In [16], Hu et al. combined a Gaussian kernel function with the Euclidean distance and generated fuzzy information granules. The Euclidean distance cannot deal with hybrid attributes directly. Therefore, we replace the Euclidean distance with the HD-I distance and construct fuzzy information granules.

Let $U$ be a finite universe and $U \neq \emptyset$. The samples are $m$-dimension vectors. Moreover, $\forall x_{i}, x_{k} \in U, x_{i}=<$ $x_{i 1}, x_{i 2}, \ldots, x_{i m}>, x_{k}=<x_{k 1}, x_{k 2}, \ldots, x_{k m}>$. The Gaussian kernel function

$$
k\left(x_{i}, x_{k}\right)=\exp \left(-\frac{\left\|x_{i}-x_{k}\right\|^{2}}{2 \delta^{2}}\right)
$$

can be used to compute the similarity between samples $x_{i}$ and $x_{k}$. $\left\|x_{i}-x_{k}\right\|$ is the HD-I distance between $x_{i}$ and $x_{k}$. The following results hold:

(1) $k\left(x_{i}, x_{k}\right) \in[0,1]$,

(2) $k\left(x_{i}, x_{k}\right)=k\left(x_{k}, x_{i}\right)$,

(3) $k\left(x_{i}, x_{i}\right)=1$.

Theorem 1. [2] Gaussian kernel $k: U \times U \rightarrow[0,1]$ satisfies $T_{\cos }$-transitivity.

The fuzzy relation $R_{H}$ computed with Formula (15) is a $T_{c o s}$-equivalence relation [16].

Example 4. Based on Table 1, we use a Gaussian kernel function to compute the $T_{\text {cos }}$-equivalence relation $R_{H}$. Let $\delta^{2}=0.8$. Each sample is a seven-dimensional vector. The fuzzy relation between each two samples can be computed by Equation (15). For example, $R_{H}\left(x_{1}, x_{3}\right)=k\left(x_{1}, x_{3}\right)=\exp \left(-\frac{H D-I^{2}\left(x_{1}, x_{3}\right)}{2 \times 0.8}\right)=\exp \left(-1.74^{2} / 1.6\right)=0.15$. Therefore, 


$$
R_{H}=\left(\begin{array}{ccccccccc}
1 & 0.91 & 0.15 & 0.31 & 0.15 & 0.28 & 0.07 & 0.52 & 0.21 \\
& 1 & 0.16 & 0.26 & 0.10 & 0.23 & 0.05 & 0.48 & 0.18 \\
& & 1 & 0.08 & 0.19 & 0.18 & 0.10 & 0.10 & 0.14 \\
& & & 1 & 0.43 & 0.06 & 0.03 & 0.15 & 0.06 \\
& & & & 1 & 0.08 & 0.04 & 0.10 & 0.08 \\
& & & & & 1 & 0.15 & 0.31 & 0.49 \\
& & & & & & 1 & 0.06 & 0.11 \\
& & & & & & & 1 & 0.10 \\
& & & & & & & & 1
\end{array}\right)
$$

Given a hybrid attribute set $B \subseteq C$, the algorithm for generating fuzzy information granules is designed as follows (see Algorithm 1).

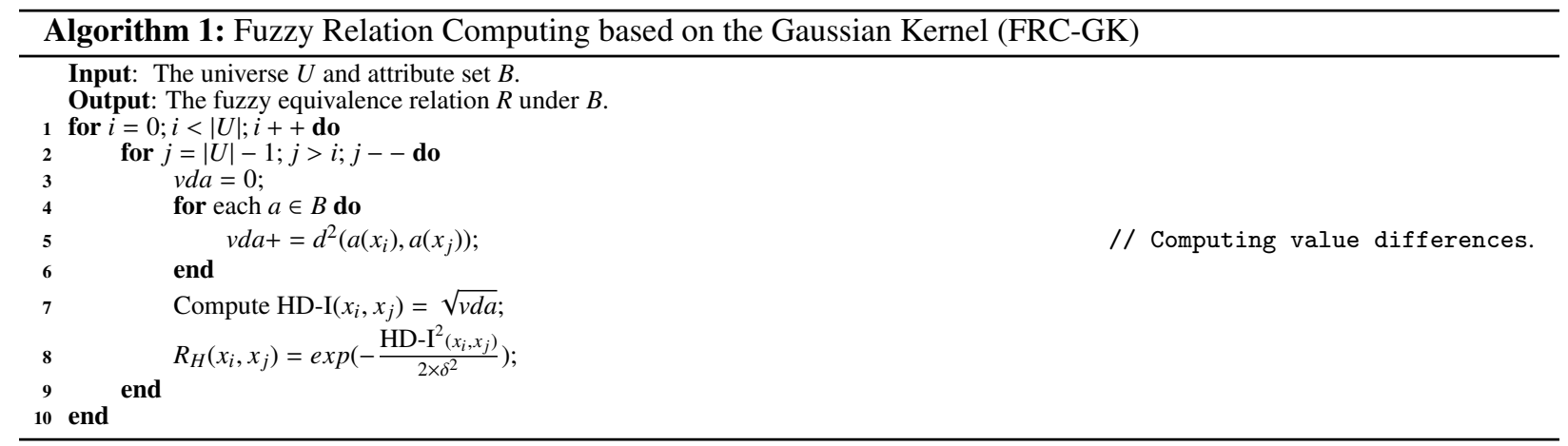

The time complexity of Algorithm 1 is $\mathrm{O}\left(m \times n^{2}\right)$, where $m$ and $n$ are the numbers of features and samples, respectively.

\subsection{Gaussian kernelized FRS}

Definition 18. [15] Let $H I S=<U, C \cup D, V, f>, R_{G}$ be a Gaussian Kernelized $T$-fuzzy equivalence relation on $U$, and $\tilde{X}$ be a fuzzy subset of $U$. The fuzzy lower and upper approximations of $\tilde{X}$ related to $R_{G}$ are defined by

(1) $S$ - Gaussian fuzzy lower approximation operator: $\underline{R}_{G S} \tilde{X}(x)=\inf _{y \in U} S\left(N\left(R_{G}(x, y)\right), \tilde{X}(y)\right)$,

(2) $\theta$-Gaussian fuzzy lower approximation operator : $R_{G \theta} \tilde{X}(x)=\inf _{y \in U} \theta_{T}\left(R_{G}(x, y), \tilde{X}(y)\right)$,

(3) $T$ - Gaussian fuzzy upper approximation operator: $\overline{\overline{R_{G T}}} \tilde{X}(x)=\sup _{y \in U} T\left(R_{G}(x, y), \tilde{X}(y)\right)$,

(4) $\sigma$-Gaussian fuzzy upper approximation operator: $\overline{R_{G \sigma}} \tilde{X}(x)=\sup _{y \in U} \sigma\left(N\left(R_{G}(x, y)\right), \tilde{X}(y)\right)$.

In this work, we focus on the definitions of $R_{G \theta} \tilde{X}$ and $\overline{R_{G T}} \tilde{X}$. Since $T_{c o s}$ is a special $T$-norm, based on Definition 18 , the following definition can be obtained.

Definition 19. Let $H I S=\left\langle U, C \cup D, V, f>, R_{H}\right.$ be a Gaussian Kernelized $T_{\cos }$-fuzzy equivalence relation on $U$, and $\tilde{X}$ be a fuzzy subset of $U$. The fuzzy lower and upper approximations of $\tilde{X}$ related to $R_{H}$ are defined by

$$
\left\{\begin{array}{l}
R_{H} \tilde{X}(x)=\inf _{y \in U} \theta_{T_{c o s}}\left(R_{H}(x, y), \tilde{X}(y)\right), \\
\overline{\overline{R_{H}}} \tilde{X}(x)=\sup _{y \in U} T_{c o s}\left(R_{H}(x, y), \tilde{X}(y)\right),
\end{array}\right.
$$

where 
(1) $R_{H}(x, y)=\exp \left(-\frac{H D-I^{2}(x, y)}{2 \times \delta^{2}}\right)$

(2) $\theta_{T_{\cos }}\left(R_{H}(x, y), \tilde{X}(y)\right)= \begin{cases}1, & R_{H}(x, y) \leq \tilde{X}(y), \\ R_{H}(x, y) \times \tilde{X}(y)+\sqrt{1-R_{H}^{2}(x, y)} \sqrt{1-\tilde{X}^{2}(y)}, & R_{H}(x, y)>\tilde{X}(y),\end{cases}$

(3) $T_{\cos }\left(R_{H}(x, y), \tilde{X}(y)\right)=\max \left\{R_{H}(x, y) \times \tilde{X}(y)-\sqrt{1-R_{H}^{2}(x, y)} \sqrt{1-\tilde{X}^{2}(y)}, 0\right\}$.

Let $\mathrm{HIS}=<U, C \cup D, V, f>$ and $U / D=\left\{d_{i}\right\}, i=1,2, \ldots,|U / D|$. The following relationship holds: $\forall x \in U$, if $x \in d_{i}, d_{i}(x)=1$; otherwise, $d_{i}(x)=0$. Therefore, we can approximate the decision regions with the fuzzy granules induced by a Gaussian function [15].

Proposition 2. For $H I S=<U, C \cup D, V, f>, \forall d_{i} \in U / D$,

$$
\left\{\begin{array}{l}
R_{H} d_{i}(x)=\inf _{y \notin d_{i}} \sqrt{1-R_{H}^{2}(x, y)}, \\
\overline{R_{H}} d_{i}(x)=\sup _{y \in d_{i}} R_{H}(x, y) .
\end{array}\right.
$$

In order to simplify the calculation, we generate the fuzzy lower and upper approximations as follows.

Proposition 3. For $H I S=<U, C \cup D, V, f>, \forall x \in U, \forall d_{i} \in U / D$,

$$
\left\{\begin{array}{l}
\frac{R_{H}}{\overline{R_{H}}} d_{i}(x)=\sqrt{1-\left(\sup _{y \notin d_{i}} R_{H}(x, y)\right)^{2}} \\
\sup _{y \in d_{i}} R_{H}(x, y)
\end{array}\right.
$$

Notably, it is clear that $y=\sqrt{1-x^{2}}, x \in[0,1]$ is a monotonically decreasing function. Because $R_{H}(x, y) \in[0,1]$, $\sqrt{1-\left(\sup \left(R_{H}(x, y)\right)\right)^{2}}=\inf \left(\sqrt{1-R_{H}^{2}(x, y)}\right)$ holds. Therefore, $\underline{R}_{H} d_{i}(x)=\sqrt{1-\left(\sup _{y \notin d_{i}} R_{H}(x, y)\right)^{2}}$.

Example 5. Based on Examples 1 and $4, U / D=\left\{d_{1}, d_{2}, d_{3}\right\}, d_{1}=\left\{x_{1}, x_{2}, x_{3}, x_{8}\right\}, d_{2}=\left\{x_{4}, x_{5}\right\}$, and $d_{3}=\left\{x_{6}, x_{7}, x_{9}\right\}$. According to Proposition 3,

$$
\begin{aligned}
\underline{R_{H}} d_{1} & \left(x_{1}\right)=\sqrt{1-\left(\sup _{y \notin d_{1}} R_{H}\left(x_{1}, y\right)\right)^{2}} \\
& =\sqrt{1-\left(\sup \left\{R_{H}\left(x_{1}, x_{4}\right), R_{H}\left(x_{1}, x_{5}\right), R_{H}\left(x_{1}, x_{6}\right), R_{H}\left(x_{1}, x_{7}\right), R_{H}\left(x_{1}, x_{9}\right)\right\}\right)^{2}} \\
& =\sqrt{1-(\sup \{0.31,0.15,0.28,0.07,0.21\})^{2}} \\
& =0.95 .
\end{aligned}
$$

Similarly, we have,

$R_{H} d_{1}=\left\{0.95 / x_{1}, 0.97 / x_{2}, 0.98 / x_{3}, 0 / x_{4}, 0 / x_{5}, 0 / x_{6}, 0 / x_{7}, 0.95 / x_{8}, 0 / x_{9}\right\}$.

$\overline{R_{H}} d_{2}=\left\{0 / x_{1}, 0 / x_{2}, 0 / x_{3}, 0.95 / x_{4}, 0.98 / x_{5}, 0 / x_{6}, 0 / x_{7}, 0 / x_{8}, 0 / x_{9}\right\}$.

$\overline{R_{H}} d_{3}=\left\{0 / x_{1}, 0 / x_{2}, 0 / x_{3}, 0 / x_{4}, 0 / x_{5}, 0.95 / x_{6}, 0.99 / x_{7}, 0 / x_{8}, 0.98 / x_{9}\right\}$.

$\overline{\overline{R_{H}}} d_{1}\left(x_{1}\right)=\sup _{y \in d_{1}} R_{H}\left(x_{1}, y\right)=\sup \left\{R_{H}\left(x_{1}, x_{1}\right), R_{H}\left(x_{1}, x_{2}\right), R_{H}\left(x_{1}, x_{3}\right), R_{H}\left(x_{1}, x_{8}\right)\right\}=\sup \{1,0.91,0.15,0.52\}=1$.

Likewise, the other upper approximations can be computed. Therefore, we have

$\overline{R_{H}} d_{1}=\left\{1 / x_{1}, 1 / x_{2}, 1 / x_{3}, 0.31 / x_{4}, 0.19 / x_{5}, 0.31 / x_{6}, 0.10 / x_{7}, 1 / x_{8}, 0.21 / x_{9}\right\}$.

$\overline{R_{H}} d_{2}=\left\{0.31 / x_{1}, 0.26 / x_{2}, 0.19 / x_{3}, 1 / x_{4}, 1 / x_{5}, 0.08 / x_{6}, 0.05 / x_{7}, 0.15 / x_{8}, 0.08 / x_{9}\right\}$.

$\overline{R_{H}} d_{3}=\left\{0.28 / x_{1}, 0.23 / x_{2}, 0.18 / x_{3}, 0.06 / x_{4}, 0.08 / x_{5}, 1 / x_{6}, 1 / x_{7}, 0.31 / x_{8}, 1 / x_{9}\right\}$.

\section{A non-incremental approach for updating approximations based on FRS under the variation of attribute values}

Given an HIS $=<U, C \cup D, V, f>$ at time $t, U \neq \emptyset$ and $C \cap D=\emptyset$. Suppose there are some attribute values that vary at time $t+1$. The fuzzy equivalence relations will be changed. Moreover, the membership degree of the lower and upper approximations will be changed accordingly. Let $R_{H}^{(t)}$ be the fuzzy equivalence relation at time $t$. For each fuzzy set $\tilde{X} \subseteq U$, the fuzzy lower and upper approximations are denoted by $\underline{R_{H}^{(t)}} \tilde{X}$ and $\overline{R_{H}^{(t)}} \tilde{X}$ at time $t$, respectively. Let HIS' 
$=\left\langle U^{\prime}, C^{\prime} \cup D^{\prime}, V^{\prime}, f^{\prime}>\right.$ at time $t+1$ and $U^{\sim}$ be the changing object set. The fuzzy lower and upper approximations of $\tilde{X}$ are denoted by $\underline{R_{H}^{(t+1)}} \tilde{X}$ and $\overline{R_{H}^{(t+1)}} \tilde{X}$, respectively.

We design Non-Incremental Updating Approximations based on Fuzzy Rough Sets (NIUAFRS) to deal with the dynamic HIS. When select attribute values vary, the NIUAFRS algorithm computes all of the fuzzy relations. Then, according to Proposition 3, the lower and upper approximations are recomputed. The detailed process procedure is presented in Algorithm 2.

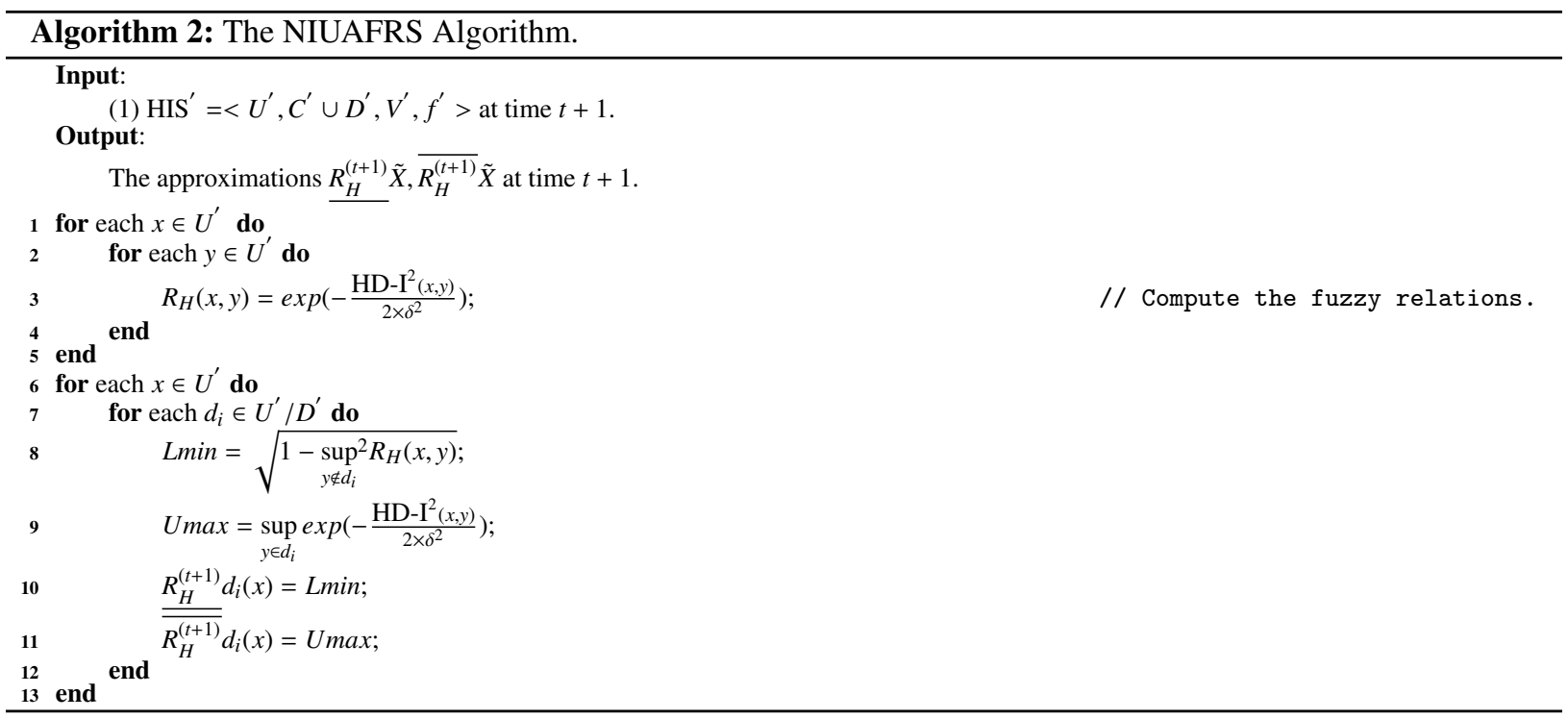

In Algorithm 2, Lines 1-5 compute the fuzzy relations. Let $m=\left|C^{\prime}\right|$ be the total number of attributes, $n=|U|$ be the total number of objects in $U$, and $l=U / D^{\prime}$ be the total number of decision classes. Because any two objects are required to calculate the fuzzy binary relation, the time complexity is $O\left(m \times n^{2}\right)$. Lines 6-13 compute the lower and upper approximations. There are two supremum functions and the time complexity is $O(\log n)$. Because each object must compute the membership degree of each decision, the total time complexity is $O(m \times n \times l \times \log n)$. In general, $l<<n, O(m \times n \times l \times \log n)<O\left(m \times n^{2}\right)$. Therefore, the time complexity of NIUAFRS is $O\left(m \times n^{2}\right)$.

\section{An FRS approach of updating approximations under the variation of the attribute values}

The variation of attribute values consists of two cases: the variation of condition attribute values and decision attribute values.

\subsection{The variation of condition attribute values}

In HIS, condition attributes are hybrid, and decision attributes often are categorical. The variation of condition attribute values does not influence the decision partition. Therefore, according to the changes of condition attribute values, there are two cases of decision classes:

(1) No condition attribute values are changed,

(2) Some condition attribute values are modified.

Suppose there are objects $U^{\sim}=\left\{x_{1}^{\sim}, \ldots, x_{m}^{\sim}\right\}$ whose attribute values are changed in the HIS at time $t+1$, where $U^{\sim} \subseteq U$. Note that $U / D=\left\{d_{l_{1}}, \ldots, d_{l_{p}}, d_{k_{1}}^{\sim}, \ldots, d_{k_{q}}^{\sim}\right\}$, where $d_{l_{1}}, \ldots, d_{l_{p}}$ denote decision classes whose objects' attribute values remain unchanged; $d_{k_{1}}^{\sim}, \ldots, d_{k_{q}}^{\sim}$ denote the decision classes that some objects' attribute values are changed.

The fuzzy relations may be enlarged or lessened with the variation of condition attribute values.

Definition 20. Given $H I S=<U, C \cup D, V, f>$, let $R_{H}^{(t)}$ and $R_{H}^{(t+1)}$ be the fuzzy equivalence relation at time $t$ and $t+1$, respectively. $\forall x, y \in U$ for which the attribute values of $x$ or y are changed,

(1) $R_{H}^{\wedge}(x, y)$ denotes the fuzzy relation enlargement if $R_{H}^{(t+1)}(x, y)>R_{H}^{(t)}(x, y)$.

(2) $R_{H}^{\vee}(x, y)$ denotes the fuzzy relation lessening if $R_{H}^{(t+1)}(x, y)<R_{H}^{(t)}(x, y)$. 
The unchanging decision classes $\left\{d_{l_{1}}, \ldots, d_{l_{p}}\right\}$ give rise to the following propositions.

Proposition 4. $\forall d_{i} \in\left\{d_{l_{1}}, \ldots, d_{l_{p}}\right\}$, the following results hold for the update of lower approximations:

(1) $\forall x \in d_{i}$,

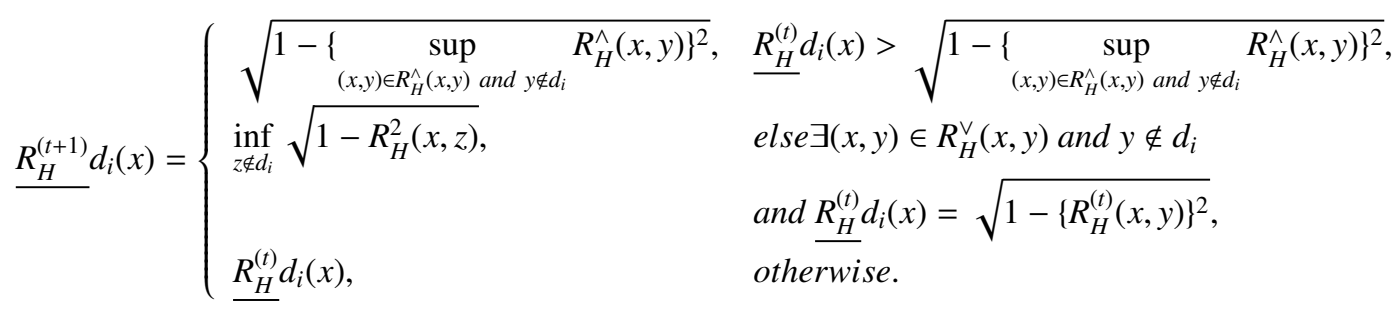

(2) $\forall x \notin d_{i}$,

$$
\underline{R_{H}^{(t+1)}} d_{i}(x)=\underline{R_{H}^{(t)}} d_{i}(x)=0 .
$$

\section{Proof 1.}

(1) $\forall d_{i} \in\left\{d_{l_{1}}, \ldots, d_{l_{p}}\right\}$ and $\forall x \in d_{i}$.

Because $\underline{R_{H}^{(t)}} d_{i}(x)=\inf _{y \notin d_{i}} \sqrt{1-R_{H}^{2}(x, y)}$, if $R_{H}(x, y)$ increases, $\underline{R_{H}^{(t)}} d_{i}(x)$ possibly decreases; if $R_{H}(x, y)$ decreases, $R_{H}^{(t)} d_{i}(x)$ does not decrease.

$$
\begin{aligned}
\underline{R_{H}^{(t+1)} d_{i}(x)} & =\inf _{y \notin d_{i}} \sqrt{1-R_{H}^{2}(x, y)} \\
& =\inf \left\{\inf _{y \notin d_{i} \text { and }(x, y) \in R_{H}^{\wedge}(x, y)} \sqrt{1-\left(R_{H}^{\wedge}(x, y)\right)^{2}}, \inf _{y \notin d_{i} \text { and }(x, y) \notin R_{H}^{\wedge}(x, y)} \sqrt{1-R_{H}^{2}(x, y)}\right\} \\
& =\inf \left\{\sqrt{1-\left(\sup _{y \notin d_{i} \text { and }(x, y) \in R_{H}^{\wedge}(x, y)} R_{H}^{\wedge}(x, y)\right)^{2}}, \inf _{y \notin d_{i} \text { and }(x, y) \notin R_{H}^{\wedge}(x, y)} \sqrt{1-R_{H}^{2}(x, y)}\right\} .
\end{aligned}
$$

Because $\inf _{y \notin d_{i} \text { and }(x, y) \notin R_{H}^{\wedge}(x, y)} \sqrt{1-R_{H}^{2}(x, y)} \geq \underline{R_{H}^{(t)}} d_{i}(x)$, if $\underline{R_{H}^{(t)}} d_{i}(x)>\sqrt{1-\left(\sup _{y \notin d_{i} \text { and }(x, y) \in R_{H}^{\wedge}(x, y)} R_{H}^{\wedge}(x, y)\right)^{2}}$, it is clear that

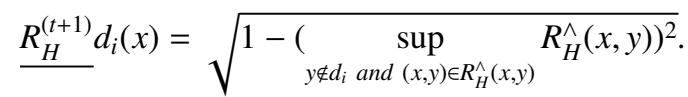

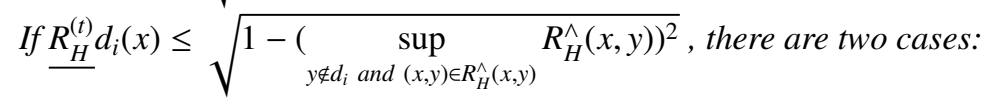

- $\exists(x, y) \in R_{H}^{\vee}(x, y)$ and $y \notin d_{i}$ and $\underline{R_{H}^{(t)}} d_{i}(x)=\sqrt{1-\left\{R_{H}^{(t)}(x, y)\right\}^{2}}$,

- $\nexists(x, y) \in R_{H}^{\vee}(x, y)$ and $y \notin d_{i}$ and $\underline{R_{H}^{(t)}} d_{i}(x)=\sqrt{1-\left\{R_{H}^{(t)}(x, y)\right\}^{2}}$.

For the first case, the lower approximation of $R_{H}^{(t)} d_{i}(x)$ increases. Therefore, the lower approximations of $d_{i}$ must be recomputed. According to Proposition 2,

$\underline{R_{H}^{(t+1)}} d_{i}(x)=\inf _{y \notin d_{i}} \sqrt{1-R_{H}^{2}(x, y)}$.

For the second case, the lower approximations do not change although some fuzzy relations are altered. Therefore,

$\underline{R_{H}^{(t+1)}} d_{i}(x)=\underline{R_{H}^{(t)}} d_{i}(x)$ 
(2) $\forall d_{i} \in\left\{d_{l_{1}}, \ldots, d_{l_{p}}\right\}$ and $\forall x \notin d_{i}$.

$\underline{R_{H}^{(t)}} d_{i}(x)=\inf _{y \notin d_{i}} \sqrt{1-R_{H}^{2}(x, y)}=\sqrt{1-\left\{\sup _{y \notin d_{i}} R_{H}(x, y)\right\}^{2}}=\sqrt{1-\left\{R_{H}(x, x)\right\}^{2}}=\sqrt{1-1^{2}}=0$.

Note that $R_{H}(x, x)$ is 1; therefore,

$\underline{R_{H}^{(t+1)}} d_{i}(x)=\underline{R_{H}^{(t)}} d_{i}(x)=0$.

Proposition 5. $\forall d_{i} \in\left\{d_{l_{1}}, \ldots, d_{l_{p}}\right\}$, the following results hold for the update of upper approximations.

(1) $\forall x \in d_{i}$ or $x \notin U^{\sim}$,

$$
\overline{R_{H}^{(t+1)}} d_{i}(x)=\overline{R_{H}^{(t)}} d_{i}(x)
$$

(2) $\forall x \notin d_{i}$ and $x \in U^{\sim}$,

$$
\overline{R_{H}^{(t+1)}} d_{i}(x)= \begin{cases}\sup _{(x, y) \in R_{H}^{\wedge}(x, y) \text { and } y \in d_{i}} R_{H}^{\wedge}(x, y), & \overline{R_{H}^{(t)}} d_{i}(x)<\sup _{(x, y) \in R_{H}^{\wedge}(x, y) \text { and } y \in d_{i}} R_{H}^{\wedge}(x, y), \\ \sup _{z \in d_{i}} R_{H}(x, z), & \text { else } \exists(x, y) \in R_{H}^{\vee}(x, y) \text { and } y \in d_{i} \text { and } \overline{R_{H}^{(t)}} d_{i}(x)=R_{H}^{(t)}(x, y), \\ \underline{R_{H}^{(t)}} d_{i}(x), & \text { otherwise. }\end{cases}
$$

\section{Proof 2.}

(1) $\forall d_{i} \in\left\{d_{l_{1}}, \ldots, d_{l_{p}}\right\}, \forall x \in d_{i}$, or $x \notin U^{\sim}$.

$\forall x \in d_{i}$, because $\overline{R_{H}} d_{i}(x)=\sup _{y \in d_{i}} R_{H}(x, y)=\sup _{y \in d_{i}} R_{H}(x, x)=1$ is a constant, $\overline{R_{H}^{(t+1)}} d_{i}(x)=\overline{R_{H}^{(t)}} d_{i}(x)=1$.

When $x \notin U^{\sim}, \overline{R_{H}^{(t+1)}} d_{i}(x)=\sup _{y \in d_{i}} R_{H}^{(t+1)}(x, y)=\sup _{y \in d_{i}} R_{\text {and }} R_{y}^{(t+1)}(x, y)=\sup _{y \in d_{i}} R_{H}^{(t)}(x, y)=\overline{R_{H}^{(t)}} d_{i}(x)$.

(2) $\forall x \notin d_{i}$ and $x \in U^{\sim}$,

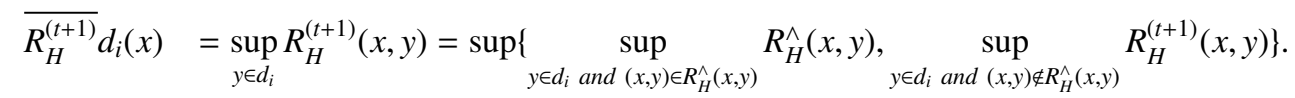

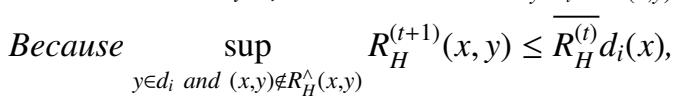

if $\overline{R_{H}^{(t)}} d_{i}(x)<\sup _{y \in d_{i} \text { and }(x, y) \in R_{H}^{\wedge}(x, y)} R_{H}^{\wedge}(x, y)$, it is clear that

$\overline{R_{H}^{(t+1)}} d_{i}(x)=\sup _{y \in d_{i} \text { and }(x, y) \in R_{H}^{\wedge}(x, y)} R_{H}^{\wedge}(x, y)$.

If $\overline{R_{H}^{(t)}} d_{i}(x) \geq \sup _{y \in d_{i} \text { and }(x, y) \in R_{H}^{\wedge}(x, y)} R_{H}^{\wedge}(x, y)$, there are two cases:

- $\exists(x, y) \in R_{H}^{\vee}(x, y)$ and $y \in d_{i}$ and $\overline{R_{H}^{(t)}} d_{i}(x)=R_{H}^{(t)}(x, y)$,

- $\nexists(x, y) \in R_{H}^{\vee}(x, y)$ and $y \in d_{i}$ and $\overline{R_{H}^{(t)}} d_{i}(x)=R_{H}^{(t)}(x, y)$.

For the first case, the upper approximation of $\overline{R_{H}^{(t)}} d_{i}(x)$ will decrease. Therefore, the upper approximations of $d_{i}$ must be recomputed. According to Proposition 2, 


$$
\overline{R_{H}^{(t+1)}} d_{i}(x)=\sup _{y \in d_{i}} R_{H}(x, y)
$$

For the second case, the upper approximations do not change although some equivalence relations are changed. Therefore,

$$
\overline{R_{H}^{(t+1)}} d_{i}(x)=\overline{R_{H}^{(t)}} d_{i}(x)
$$

With respect to the decision classes $\left\{d_{k_{1}}^{\sim}, \ldots, d_{k_{q}}^{\sim}\right\}$, where some of the condition attribute values are varied, the lower and upper approximations can be updated incrementally according to the following propositions.

Proposition 6. $\forall d_{i} \in\left\{d_{k_{1}}^{\sim}, \ldots, d_{k_{q}}^{\sim}\right\}$, the following results hold for the update of lower approximations.

(1) $\forall x \notin d_{i}$,

$$
\underline{R_{H}^{(t+1)}} d_{i}(x)=\underline{R_{H}^{(t)}} d_{i}(x)=0 .
$$

(2) $\forall x \in d_{i}$,

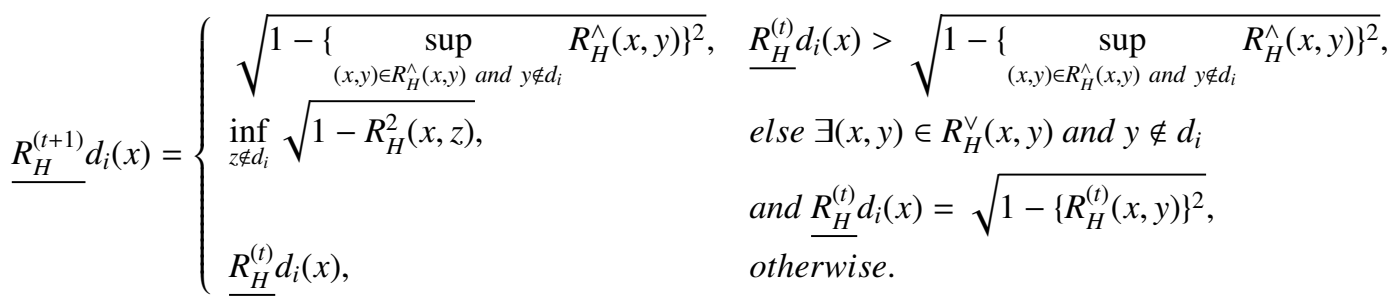

Proof 3. The proof follows in the same way as Proposition 4.

Proposition 7. $\forall d_{i} \in\left\{d_{k_{1}}^{\sim}, \ldots, d_{k_{q}}^{\sim}\right\}$, the following results hold for the update of upper approximations:

(1) $\forall x \in d_{i}$,

$$
\overline{R_{H}^{(t+1)}} d_{i}(x)=\overline{R_{H}^{(t)}} d_{i}(x)=1 .
$$

(2) $\forall x \notin d_{i}$,

$$
\overline{R_{H}^{(t+1)}} d_{i}(x)= \begin{cases}\sup _{(x, y) \in R_{H}^{\wedge}(x, y) \text { and } y \in d_{i}} R_{H}^{\wedge}(x, y), & \overline{R_{H}^{(t)}} d_{i}(x)<\sup _{(x, y) \in R_{H}^{\wedge}(x, y) \text { and } y \in d_{i}} R_{H}^{\wedge}(x, y), \\ \sup _{z \in d_{i}} R_{H}(x, z), & \text { else } \exists(x, y) \in R_{H}^{\vee}(x, y) \text { and } y \in d_{i} \text { and } \overline{R_{H}^{(t)}} d_{i}(x)=R_{H}^{(t)}(x, y), \\ \frac{R_{H}^{(t)}}{d_{i}(x),} & \text { otherwise. }\end{cases}
$$

Proof 4. The proof follows in the same way as Proposition 5.

Example 6. As shown Table 2, and based on Example 1, the attribute values $a_{3}\left(x_{6}\right)$ and $a_{1}\left(x_{9}\right)$ are changed. At time $t, U / D=\left\{d_{1}, d_{2}, d_{3}\right\}, d_{1}=\left\{x_{1}, x_{2}, x_{3}, x_{8}\right\}, d_{2}=\left\{x_{4}, x_{5}\right\}$, and $d_{3}=\left\{x_{6}, x_{7}, x_{9}\right\}$.

At time $t+1, U^{\sim}=\left\{x_{1}^{\sim}, x_{2}^{\sim}\right\}=\left\{x_{6}, x_{9}\right\}$. Decisions $d_{l_{1}}=d_{1}$ and $d_{l_{2}}=d_{2}$ are unchanged, and decision $d_{k_{1}}^{\sim}=d_{3}$ is changed. At time t, according to Example 4, 
Table 2: The variation of condition attribute values in HIS

\begin{tabular}{|c|c|c|c|c|c|c|c|c|}
\hline$U$ & $\begin{array}{c}\text { Urinary } \\
\text { Color }\left(a_{1}\right) \\
\end{array}$ & $\begin{array}{c}\text { Urinary } \\
\text { Tuberculosis }\left(a_{2}\right)\end{array}$ & $\begin{array}{l}\mathbf{P H} \\
\left(a_{3}\right) \\
\end{array}$ & $\begin{array}{c}\text { Urinary } \\
\text { Irritation }\left(a_{4}\right)\end{array}$ & $\begin{array}{l}\mathbf{R B C} \\
\left(a_{5}\right) \\
\end{array}$ & $\begin{array}{c}\mathbf{W B C} \\
\left(a_{6}\right) \\
\end{array}$ & $\begin{array}{l}\mathrm{CT} \\
\left(a_{7}\right)\end{array}$ & $d$ \\
\hline$x_{1}$ & Pink & - & 4.0 & $\{\mathrm{M}, \mathrm{U}, \mathrm{P}\}$ & {$[1,4]$} & {$[3,7]$} & & Nephritis \\
\hline$x_{2}$ & Pink & - & 4.5 & $\{\mathrm{M}, \mathrm{U}, \mathrm{P}\}$ & {$[2,4]$} & {$[2,6]$} & & Nephritis \\
\hline$x_{3}$ & Red & $?$ & 3.8 & $\{\mathrm{M}\}$ & {$[1,3]$} & {$[1,4]$} & & Nephritis \\
\hline$x_{4}$ & Pink & + & 3.6 & $\{\mathrm{U}\}$ & {$[2,5]$} & {$[5,7]$} & & rotuberculosis \\
\hline$x_{5}$ & Red & + & 3.5 & $\{\mathrm{U}\}$ & {$[1,4]$} & {$[6,7]$} & & rotuberculosis \\
\hline$x_{6}$ & Yellow & - & $5.8(5.5)$ & $\{\mathrm{U}, \mathrm{P}\}$ & {$[0,2]$} & {$[0,5]$} & & Health \\
\hline$x_{7}$ & Yellow & $?$ & $?$ & $\{\mathrm{P}\}$ & {$[0,2]$} & {$[1,4]$} & & Health \\
\hline$x_{8}$ & Yellow & - & 4.1 & $\{\mathrm{M}, \mathrm{U}, \mathrm{P}\}$ & {$[2,4]$} & {$[4,6]$} & & Nephritis \\
\hline$x_{9}$ & Pink(?) & - & 5.6 & $\{\mathrm{U}\}$ & {$[0,2]$} & {$[2,4]$} & & Health \\
\hline
\end{tabular}

$R_{H}(t)=\left(\begin{array}{ccccccccc}1 & 0.91 & 0.15 & 0.31 & 0.15 & 0.28 & 0.07 & 0.52 & 0.21 \\ & 1 & 0.16 & 0.26 & 0.10 & 0.23 & 0.05 & 0.48 & 0.18 \\ & & 1 & 0.08 & 0.19 & 0.18 & 0.10 & 0.10 & 0.14 \\ & & & 1 & 0.43 & 0.06 & 0.03 & 0.15 & 0.06 \\ & & & & 1 & 0.08 & 0.04 & 0.10 & 0.08 \\ & & & & & 1 & 0.15 & 0.31 & 0.49 \\ & & & & & & 1 & 0.06 & 0.11 \\ & & & & & & & 1 & 0.10\end{array}\right)$.

For lower approximations,

$\underline{R_{H}^{(t)}} d_{1}\left(x_{1}\right)=\sqrt{1-\left(\sup _{y \notin d_{1}} R_{H}\left(x_{1}, y\right)\right)^{2}}=\sqrt{1-(\sup \{0.31,0.15,0.28,0.07,0.21\})^{2}}=0.95$.

Similarly, the lower approximations at time t are as follows:

$R_{H}^{(t)} d_{1}=\left\{0.95 / x_{1}, 0.97 / x_{2}, 0.98 / x_{3}, 0 / x_{4}, 0 / x_{5}, 0 / x_{6}, 0 / x_{7}, 0.95 / x_{8}, 0 / x_{9}\right\}$.

$\overline{R_{H}^{(t)}} d_{2}=\left\{0 / x_{1}, 0 / x_{2}, 0 / x_{3}, 0.95 / x_{4}, 0.98 / x_{5}, 0 / x_{6}, 0 / x_{7}, 0 / x_{8}, 0 / x_{9}\right\}$.

$\overline{R_{H}^{(t)}} d_{3}=\left\{0 / x_{1}, 0 / x_{2}, 0 / x_{3}, 0 / x_{4}, 0 / x_{5}, 0.95 / x_{6}, 0.99 / x_{7}, 0 / x_{8}, 0.98 / x_{9}\right\}$

$\overline{\text { For upper approximations, }}$

$\overline{R_{H}^{(t)}} d_{1}\left(x_{1}\right)=\sup _{y \in d_{1}} R_{H}\left(x_{1}, y\right)=\sup \left\{R_{H}\left(x_{1}, x_{1}\right), R_{H}\left(x_{1}, x_{2}\right), R_{H}\left(x_{1}, x_{3}\right), R_{H}\left(x_{1}, x_{8}\right)\right\}=\sup \{1,0.91,0.15,0.52\}=1$.

Similarly, the upper approximations at time t are as follows:

$\overline{R_{H}^{(t)}} d_{1}=\left\{1 / x_{1}, 1 / x_{2}, 1 / x_{3}, 0.31 / x_{4}, 0.19 / x_{5}, 0.31 / x_{6}, 0.10 / x_{7}, 1 / x_{8}, 0.21 / x_{9}\right\}$. 


$$
\begin{aligned}
& \overline{R_{H}^{(t)}} d_{2}=\left\{0.31 / x_{1}, 0.26 / x_{2}, 0.19 / x_{3}, 1 / x_{4}, 1 / x_{5}, 0.08 / x_{6}, 0.04 / x_{7}, 0.15 / x_{8}, 0.08 / x_{9}\right\} . \\
& \overline{R_{H}^{(t)}} d_{3}=\left\{0.28 / x_{1}, 0.23 / x_{2}, 0.18 / x_{3}, 0.06 / x_{4}, 0.08 / x_{5}, 1 / x_{6}, 1 / x_{7}, 0.31 / x_{8}, 1 / x_{9}\right\} .
\end{aligned}
$$

At time $t+1$, we only need to compute the fuzzy relation between the changed objects $\left(x_{6}\right.$ and $\left.x_{9}\right)$ and the others (including the fuzzy relation between $x_{6}$ and $x_{9}$ ). The relations at time $t$ do not need to be recomputed again. Therefore,

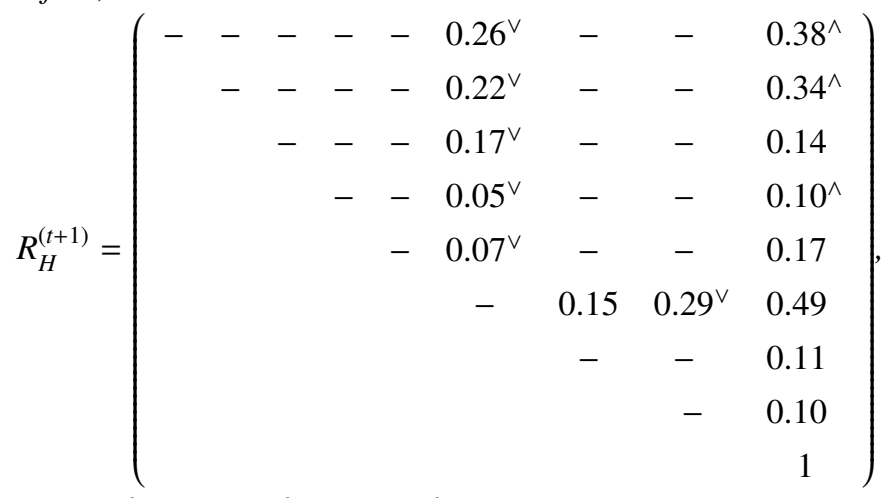

where "-" denotes unchanging relations.

(1) First, we compute lower approximations.

For decision classes $d_{l_{1}}=d_{1}$ and $d_{l_{2}}=d_{2}$, no condition attribute values are changed. According to Proposition 4 ,

$$
T=\sqrt{1-\left\{\sup _{\left(x_{1}, y\right) \in R_{H}^{\wedge}\left(x_{1}, y\right) \text { and } y \notin d_{1}} R_{H}^{\wedge}\left(x_{1}, y\right)\right\}^{2}}=\sqrt{1-\left(R_{H}^{\wedge}\left(x_{1}, x_{9}\right)\right)^{2}}=\sqrt{1-0.38^{2}}=0.92 \text {. }
$$

It is clear that $R_{H}^{(t)} d_{1}\left(x_{1}\right)=0.95>T$; therefore, $R_{H}^{(t+1)} d_{1}\left(x_{1}\right)=T=0.92$.

$$
T=\sqrt{1-\left\{\sup _{\left(x_{3}, y\right) \in R_{H}^{\vee}\left(x_{3}, y\right) \text { and } y \notin d_{1}} R_{H}^{\vee}\left(x_{3}, y\right)\right\}^{2}}=\sqrt{1-\left(R_{H}^{\vee}\left(x_{3}, x_{6}\right)\right)^{2}}=\sqrt{1-0.18^{2}}=0.9836 \text {. }
$$

It is clear that $\underline{R_{H}^{(t)}} d_{1}\left(x_{3}\right)=0.98<T$; therefore, $\underline{R_{H}^{(t+1)}} d_{1}\left(x_{3}\right)=\underline{R_{H}^{(t+1)}} d_{1}\left(x_{3}\right)=0.98$.

Similarly,

$R_{H}^{(t+1)} d_{1}=\left\{0.92 / x_{1}, 0.94 / x_{2}, 0.98 / x_{3}, 0 / x_{4}, 0 / x_{5}, 0 / x_{6}, 0 / x_{7}, 0.96 / x_{8}, 0 / x_{9}\right\}$.

$\overline{R_{H}^{(t+1)}} d_{2}=\left\{0 / x_{1}, 0 / x_{2}, 0 / x_{3}, 0.95 / x_{4}, 0.98 / x_{5}, 0 / x_{6}, 0 / x_{7}, 0 / x_{8}, 0 / x_{9}\right\}$.

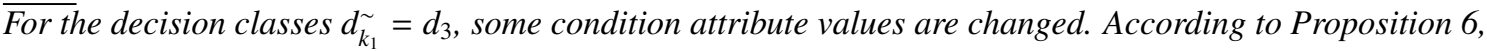

$\left(x_{1}, y\right) \in R_{H}^{\wedge}\left(x_{1}, y\right)$ and $y \notin d_{3}=\emptyset$, and $\left(x_{1}, y\right) \in R_{H}^{\vee}\left(x_{1}, y\right)$ and $y \notin d_{3}=\emptyset$. Therefore, $R_{H}^{(t+1)} d_{3}\left(x_{1}\right)=R_{H}^{(t)} d_{3}\left(x_{1}\right)$.

Similarly,

$R_{H}^{(t+1)} d_{3}=\left\{0 / x_{1}, 0 / x_{2}, 0 / x_{3}, 0 / x_{4}, 0 / x_{5}, 0.95 / x_{6}, 0.99 / x_{7}, 0 / x_{8}, 0.98 / x_{9}\right\}$.

Therefore, it follows that

$R_{H}^{(t+1)} d_{1}=\left\{0.92 / x_{1}, 0.94 / x_{2}, 0.98 / x_{3}, 0 / x_{4}, 0 / x_{5}, 0 / x_{6}, 0 / x_{7}, 0.96 / x_{8}, 0 / x_{9}\right\}$.

$\overline{R_{H}^{(t+1)}} d_{2}=\left\{0 / x_{1}, 0 / x_{2}, 0 / x_{3}, 0.95 / x_{4}, 0.98 / x_{5}, 0 / x_{6}, 0 / x_{7}, 0 / x_{8}, 0 / x_{9}\right\}$.

$\overline{R_{H}^{(t+1)}} d_{3}=\left\{0 / x_{1}, 0 / x_{2}, 0 / x_{3}, 0 / x_{4}, 0 / x_{5}, 0.95 / x_{6}, 0.99 / x_{7}, 0 / x_{8}, 0.98 / x_{9}\right\}$.

(2) Second, we compute upper approximations.

For decision classes $d_{l_{1}}=d_{1}$ and $d_{l_{2}}=d_{2}$, according to Proposition 5, $\overline{R_{H}^{(t+1)}} d_{1}\left(x_{1}\right)=\overline{R_{H}^{(t)}} d_{1}\left(x_{1}\right), \overline{R_{H}^{(t+1)}} d_{1}\left(x_{2}\right)=$ $\overline{R_{H}^{(t)}} d_{1}\left(x_{2}\right), \overline{R_{H}^{(t+1)}} d_{1}\left(x_{3}\right)=\overline{R_{H}^{(t)}} d_{1}\left(x_{3}\right), \overline{R_{H}^{(t+1)}} d_{1}\left(x_{4}\right)=\overline{R_{H}^{(t)}} d_{1}\left(x_{4}\right), \overline{R_{H}^{(t+1)}} d_{1}\left(x_{5}\right)=\overline{R_{H}^{(t)}} d_{1}\left(x_{5}\right), \overline{R_{H}^{(t+1)}} d_{1}\left(x_{7}\right)=\overline{R_{H}^{(t)}} d_{1}\left(x_{7}\right)$, and $\overline{R_{H}^{(t+1)}} d_{1}\left(x_{8}\right)=\overline{R_{H}^{(t)}} d_{1}\left(x_{8}\right)$.

For $\left(x_{6}, y\right) \in R_{H}^{\wedge}\left(x_{6}, y\right)$ and $y \in d_{1}=\emptyset, \exists\left(x_{6}, y\right) \in R_{H}^{\vee}\left(x_{6}, y\right)$ and $y \in d_{1}$ and $\overline{R_{H}^{(t)}} d_{1}\left(x_{6}\right)=R_{H}^{(t)}\left(x_{6}, y\right)$. Therefore,

$\overline{R_{H}^{(t+1)}} d_{1}\left(x_{6}\right)=\sup _{y \in d_{1}}\left\{R_{H}\left(x_{6}, y\right)\right\}=\sup \left\{, R_{H}\left(x_{1}, x_{6}\right), R_{H}\left(x_{2}, x_{6}\right), R_{H}\left(x_{3}, x_{6}\right), R_{H}\left(x_{8}, x_{6}\right)\right\}=0.29$.

$T=\sup _{\left(x_{9}, y\right) \in R_{H}^{\wedge}\left(x_{9}, y\right) \text { and } y \in d_{1}} R_{H}^{\wedge}\left(x_{9}, y\right)=\sup \left\{R_{H}\left(x_{9}, x_{1}\right), R_{H}\left(x_{9}, x_{2}\right), R_{H}\left(x_{9}, x_{3}\right)\right\}=\sup \{0.38,0.34,0.10\}=0.38>$ $\overline{R_{H}^{(t)}} d_{1}\left(x_{9}\right)$. Therefore, $\overline{R_{H}^{(t+1)}} d_{1}\left(x_{9}\right)=0.38$. 
Similarly, the upper approximations of $d_{2}$ can be computed.

$\overline{R_{H}^{(t+1)}} d_{1}=\left\{1 / x_{1}, 1 / x_{2}, 1 / x_{3}, 0.31 / x_{4}, 0.19 / x_{5}, 0.31 / x_{6}, 0.10 / x_{7}, 1 / x_{8}, 0.38 / x_{9}\right\}$.
$\overline{R_{H}^{(t+1)}} d_{2}=\left\{0.31 / x_{1}, 0.26 / x_{2}, 0.19 / x_{3}, 1 / x_{4}, 1 / x_{5}, 0.07 / x_{6}, 0.04 / x_{7}, 0.15 / x_{8}, 0.10 / x_{9}\right\}$.
For decision class $d_{k_{1}}^{\sim}=d_{3}$, some condition attribute values are changed. According to Proposition 7,

$T=\sup _{\left(x_{1}, y\right) \in R_{H}^{\wedge}\left(x_{9}, y\right) \text { and } y \in d_{3}} R_{H}^{\wedge}\left(x_{1}, y\right)=R_{H}\left(x_{1}, x_{9}\right)=0.38>\overline{R_{H}^{(t)}} d_{3}\left(x_{1}\right)$. Therefore, $\overline{R_{H}^{(t+1)}} d_{3}\left(x_{1}\right)=0.38$.

Similarly, $\overline{R_{H}^{(t+1)}} d_{3}\left(x_{2}\right)=0.34, \overline{R_{H}^{(t+1)}} d_{3}\left(x_{4}\right)=0.10$.

For $\left(x_{3}, x_{6}\right) \in R_{H}^{\wedge}\left(x_{3}, x_{6}\right)$ and $x_{6} \in d_{3}, \overline{R_{H}^{(t)}} d_{3}\left(x_{3}\right)=R_{H}^{(t)}\left(x_{3}, x_{6}\right)=0.18$. Therefore, $\overline{R_{H}^{(t+1)}} d_{3}\left(x_{3}\right)=\sup _{z \in d_{3}} R_{H}^{(t+1)}\left(x_{3}, z\right)=$ 0.17 .

Similarly, $\overline{R_{H}^{(t+1)}} d_{3}=\left\{0.38 / x_{1}, 0.34 / x_{2}, 0.17 / x_{3}, 0.10 / x_{4}, 0.08 / x_{5}, 1 / x_{6}, 1 / x_{7}, 0.29 / x_{8}, 1 / x_{9}\right\}$.

Therefore, the upper approximations are as follows:

$\overline{R_{H}^{(t+1)}} d_{1}=\left\{1 / x_{1}, 1 / x_{2}, 1 / x_{3}, 0.31 / x_{4}, 0.19 / x_{5}, 0.31 / x_{6}, 0.10 / x_{7}, 1 / x_{8}, 0.38 / x_{9}\right\}$.
$\overline{R_{H}^{(t+1)}} d_{2}=\left\{0.31 / x_{1}, 0.26 / x_{2}, 0.19 / x_{3}, 1 / x_{4}, 1 / x_{5}, 0.07 / x_{6}, 0.04 / x_{7}, 0.15 / x_{8}, 0.10 / x_{9}\right\}$.
$\overline{R_{H}^{(t+1)}} d_{3}=\left\{0.38 / x_{1}, 0.34 / x_{2}, 0.17 / x_{3}, 0.10 / x_{4}, 0.08 / x_{5}, 1 / x_{6}, 1 / x_{7}, 0.29 / x_{8}, 1 / x_{9}\right\}$.

When condition attribute values vary, the algorithm only generates the fuzzy relations between objects of $U^{\sim}$ and the others. According to the above propositions, the algorithm for Incremental Updating Approximations based on FRS under the Variation of Condition Attribute Values (IUAFRS-VCAV) is outlined in Algorithm 3.

Lines 1-7 compute the new fuzzy relations. The time complexity is $O\left(m \times n \times\left|U^{\sim}\right|\right)$, where $m=|C|$ is the total number of attributes, $n=|U|$ is the total number of objects, and $\left|U^{\sim}\right|$ is the total number of variation objects. According to Propositions 4 and 5, Lines 9-28 compute the lower approximations of the decision classes $d_{i} \in\left\{d_{l_{1}}, \ldots\right.$, $\left.d_{l_{p}}\right\}$. It is clear that the time complexity is $\max \left(O\left(\left|d_{i}\right| \times \log \left|U^{\sim}\right|\right), O\left(\left|d_{i}\right| \times \log n\right), O(n), O\left(\left|U^{\sim}\right| \times \log \left|U^{\sim}\right|\right), O\left(\left|U^{\sim}\right| \times \log \left|d_{i}\right|\right)\right)$. According to Propositions 6 and 7, Lines 29-45 compute the lower approximations of the decision classes $d_{i} \in\left\{d_{k_{1}}, \ldots\right.$, $\left.d_{k_{q}}\right\}$. It is clear that the time complexity is $\max \left(O\left(\left|d_{i}\right| \times \log n\right), O(n), O\left(m \times n \times \log \left|U^{\sim}\right|\right), O\left(\left|U^{\sim}\right| \times \log n\right)\right)$. If $\left|d_{i}\right|=n$, the time complexity of Algorithm 3 is $\max \left(O\left(m \times n \times\left|U^{\sim}\right|\right), O(n \times \log n), O\left(\left|U^{\sim}\right| \times \log n\right), O\left(\left|U^{\sim}\right| \times \log \left|U^{\sim}\right|\right)\right)$. If the total number of changing objects is $\left|U^{\sim}\right| \approx 1$, the time complexity is $O(m \times n)$; if $\left|U^{\sim}\right| \approx n$, the time complexity is $O\left(m \times n^{2}\right)$. It is clear that the incremental algorithm is superior to the non-incremental algorithm. 


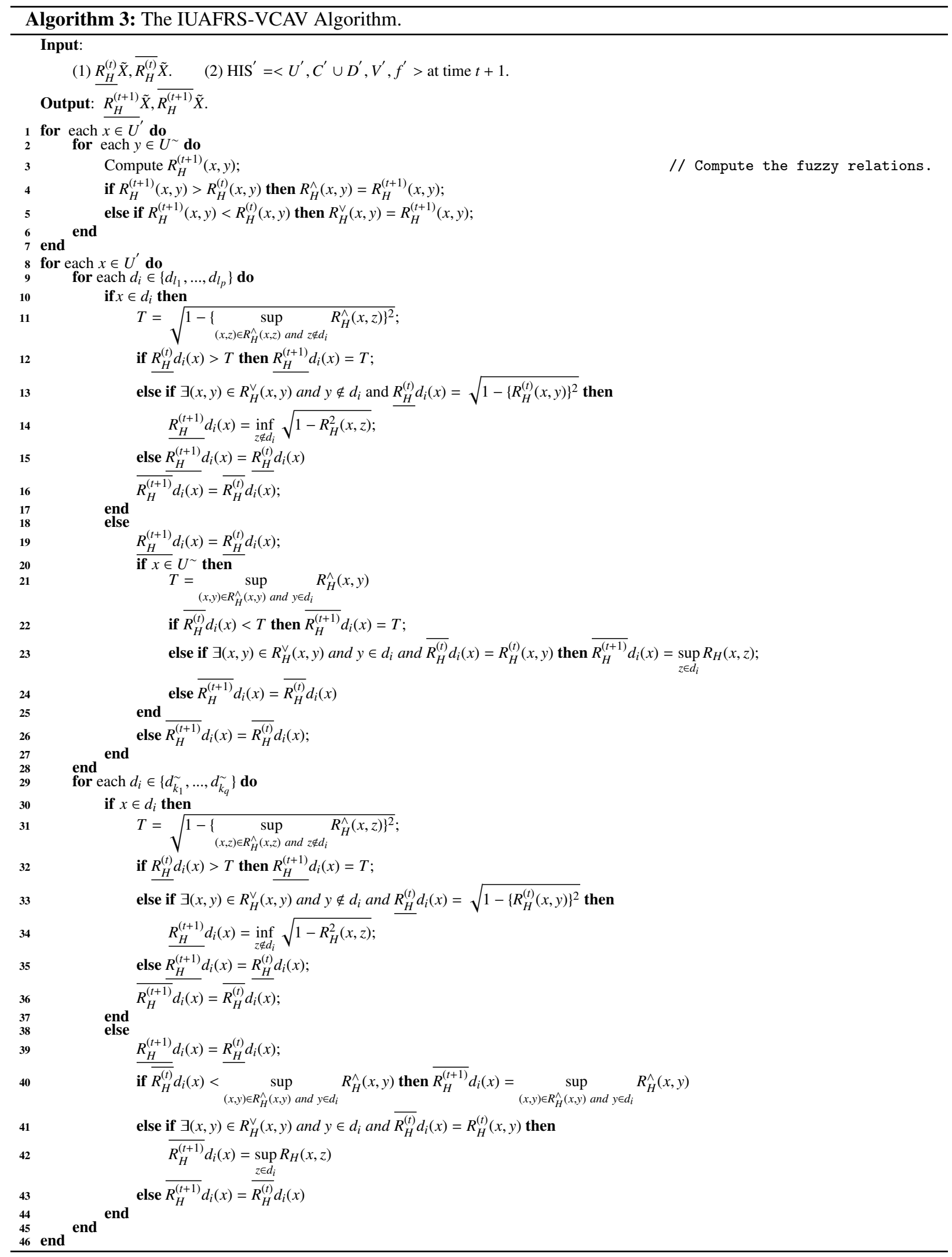




\subsection{The variation of decision attribute values}

In [8], Chen et al. presented the definition of attribute value coarsening and refining; the definitions are as follows.

Definition 21. Given HIS $=<U, A, V, f>, A=C \cup D$, and $a_{l} \in A, f\left(x_{i}, a_{l}\right)$ is the attribute value of $x_{i}$ under attribute $a_{l}$, $f\left(x_{k}, a_{l}\right)$ is the attribute value of $x_{k}(k \neq i)$ under attribute $a_{l}$, and $f\left(x_{i}, a_{l}\right) \neq f\left(x_{k}, a_{l}\right)$. Then $X_{a_{l}}=\left\{x_{i}^{\prime} \in U \mid f\left(x_{i}^{\prime}, a_{l}\right)=\right.$ $\left.f\left(x_{i}, a_{l}\right)\right\}$. Let $f\left(x_{i}^{\prime}, a_{l}\right)=f\left(x_{k}, a_{l}\right), \forall x_{i}^{\prime} \in X_{a_{l}}$. Then we say $f\left(x_{i}^{\prime}, a_{l}\right)$ is coarsened to $f\left(x_{k}, a_{l}\right)$.

Definition 22. Given HIS $=<U, A, V, f>, A=C \cup D$, and $a_{l} \in A, f\left(x_{i}, a_{l}\right)$ is the attribute value of $x_{i}$ under attribute $a_{l}$ and $X_{a_{l}}=\left\{x_{i}^{\prime} \in U \mid f\left(x_{i}^{\prime}, a_{l}\right)=f\left(x_{i}, a_{l}\right)\right\}$. Let $f\left(x_{i}^{\prime}, a_{l}\right)=v$, where $v \notin V_{l}, x_{i}^{\prime} \in X_{a_{l}}$. Then we say $f\left(x_{i}^{\prime}, a_{l}\right)$ is refined to $v$.

The variation of decision attribute values consists of two cases:

(1) Decision attribute value refining,

(2) Decision attribute value coarsening.

Given HIS $=<U, C \cup D, V, f>$ at time $t$ and $\mathrm{HIS}^{\prime}=<U^{\prime}, C^{\prime} \cup D^{\prime}, V^{\prime}, f^{\prime}>$ at time $t+1$,

$U / D=\left\{d_{u_{1}}, \ldots, d_{u_{p}}, d_{v_{1}}, \ldots, d_{v_{q}}, d_{w_{1}}^{(1)}, \ldots, d_{w_{1}}^{(k)}, \ldots, d_{w_{r}}^{(1)}, \ldots, d_{w_{r}}^{(l)}\right\}$ and

$U^{\prime} / D^{\prime}=\left\{d_{u_{1}}, \ldots, d_{u_{p}}, d_{v_{1}}^{\prime}, \ldots, d_{v_{q}}^{\prime}, d_{v_{1}}^{\vee}, \ldots, d_{v_{q}}^{\vee}, d_{w_{1}}^{\wedge}, \ldots, d_{w_{s}}^{\wedge}\right\}$.

$d_{u_{1}}, \ldots, d_{u_{p}}$ denote the decision classes in which no decision value is changed at time $t+1$.

$d_{v_{1}}, \ldots, d_{v_{q}}$ denote the decision classes at time $t$ whose attribute values are refined at time $t+1$.

$d_{v_{1}}^{\prime}, \ldots, d_{v_{q}}^{\prime}$ denote the remain decision classes being refined at time $t+1$.

$d_{v_{1}}^{\vee}, \ldots, d_{v_{q}}^{\vee}$ denote the new decision classes after refining at time $t+1$, where $\forall d_{v_{i}} \in\left\{d_{v_{1}}, \ldots, d_{v_{q}}\right\}, d_{v_{i}}=d_{v_{i}}^{\prime} \cup d_{v_{i}}^{\vee}$.

$d_{w_{1}}^{(1)}, \ldots, d_{w_{1}}^{(k)}, \ldots, d_{w_{r}}^{(1)}, \ldots, d_{w_{r}}^{(l)}$ denote the decision classes at time $t$ whose attribute values are coarsened at time $t+1$.

$d_{w_{1}}^{\wedge}, \ldots, d_{w_{s}}^{\wedge}$ denote the coarsening decision classes at time $t+1$, where $d_{w_{i}}^{\wedge}=d_{w_{i}}^{(1)} \cup \ldots \cup d_{w_{i}}^{(k)}$.

Proposition 8. $\forall d_{i} \in\left\{d_{u_{1}}, \ldots, d_{u_{p}}\right\}$, the following results hold:

$$
\left\{\begin{array}{l}
R_{H}^{(t+1)} d_{i}(x)=\underline{R_{H}^{(t)}} d_{i}(x), \\
\overline{\overline{R_{H}^{(t+1)}}} d_{i}(x)=\overline{\overline{R_{H}^{(t)}}} d_{i}(x) .
\end{array}\right.
$$

Proof 5. $\forall d_{i} \in\left\{d_{u_{1}}, \ldots, d_{u_{p}}\right\}$, the objects in these classes are unchanged at time $t+1$. Therefore, according to Proposition 2,

$$
\overline{R_{H}^{(t+1)}} d_{i}(x)=\overline{R_{H}^{(t)}} d_{i}(x)=\sup _{y \in d_{i}} R_{H}(x, y) \text {. }
$$

Because the objects of $U$ are not changed at time $t+1$, the objects of $U-d_{i}$ are not changed. Therefore, according to Proposition 2,

$$
\underline{R_{H}^{(t+1)}} d_{i}(x)=\underline{R_{H}^{(t)}} d_{i}(x)=\inf _{y \notin d_{i}} \sqrt{1-R_{H}^{2}(x, y)} .
$$

With respect to the first changing case, there are some decision classes in which some decision values are refined.

Proposition 9. Let $d_{v_{1}}, \ldots, d_{v_{q}}$ be refined to $\left\{d_{v_{1}}^{\prime}, d_{v_{1}}^{\vee}\right\}, \ldots,\left\{d_{v_{q}}^{\prime}, d_{v_{q}}^{\vee}\right\}$ at time $t+1$, respectively, where $d_{v_{i}}=d_{v_{i}}^{\prime} \cup d_{v_{i}}^{\vee}$. The following results hold for the update of lower and upper approximations:

(1) $\forall d_{v_{i}}^{\prime} \in\left\{d_{v_{1}}^{\prime}, \ldots, d_{v_{q}}^{\prime}\right\}$,

$$
\begin{aligned}
& \underline{R_{H}^{(t+1)}} d_{v_{i}}^{\prime}(x)=\inf \left\{\underline{\underline{R_{H}^{(t)}}} d_{v_{i}}(x), \inf _{y \in d_{v_{i}}} \sqrt{\left.1-R_{H}^{2}(x, y)\right\}},\right. \\
& \overline{R_{H}^{(t+1)}} d_{v_{i}}^{\prime}(x)= \begin{cases}\overline{R_{H}^{(t)}} d_{v_{i}}(x), & \overline{R_{H}^{(t)}} d_{v_{i}}(x)>\sup _{y \in d v_{i}^{v}} R_{H}(x, y), \\
\sup _{y \in d_{v_{i}}^{\prime}} R_{H}(x, y), & \overline{R_{H}^{(t)}} d_{v_{i}}(x) \leq \sup _{y \in d_{v_{i}}^{v}} R_{H}(x, y) .\end{cases}
\end{aligned}
$$


(2) $\forall d_{v_{i}}^{\vee} \in\left\{d_{v_{1}}^{\vee}, \ldots, d_{v_{q}}^{\vee}\right\}$,

$$
\begin{aligned}
& \underline{R_{H}^{(t+1)}} d_{v_{i}}^{\vee}(x)=\inf \left\{\underline{R_{H}^{(t)}} d_{v_{i}}(x), \inf _{y \in d_{v_{i}}^{\prime}} \sqrt{\left.1-R_{H}^{2}(x, y)\right\}},\right. \\
& \overline{R_{H}^{(t+1)}} d_{v_{i}}^{\vee}(x)=\sup _{y \in d_{v_{i}}^{\vee}} R_{H}(x, y) .
\end{aligned}
$$

\section{Proof 6.}

(1) $\forall d_{v_{i}}^{\prime} \in\left\{d_{v_{1}}^{\prime}, \ldots, d_{v_{q}}^{\prime}\right\}$, let $d_{v_{i}}$ be refined to $\left\{d_{v_{i}}^{\prime}, d_{v_{i}}^{\vee}\right\}$ at time $t+1$, where $d_{v_{i}}=d_{v_{i}}^{\prime} \cup d_{v_{i}}^{\vee}$.

Because $d_{v_{i}}=d_{v_{i}}^{\prime} \cup d_{v_{i}}^{\vee}, \overline{d_{v_{i}}}=\overline{d_{v_{i}}^{\prime}} \cap \overline{d_{v_{i}}^{\vee}}$ and $\overline{d_{v_{i}}^{\prime}}=\overline{d_{v_{i}}^{\prime}} \cap \overline{d_{v_{i}}^{\vee}} \cup d_{v_{i}}^{\vee}=\overline{d_{v_{i}}} \cup d_{v_{i}}^{\vee}$. According to Proposition 2,

$\underline{R_{H}^{(t+1)}} d_{v_{i}}^{\prime}(x)=\inf _{y \notin d_{v_{i}}^{\prime}} \sqrt{1-R_{H}^{2}(x, y)}=\inf _{y \notin d_{v_{i}} \text { or }}{ }_{y \in d d_{v_{i}}^{v}} \sqrt{1-R_{H}^{2}(x, y)}=\inf \left\{\inf _{y \notin d_{v_{i}}} \sqrt{1-R_{H}^{2}(x, y)}, \inf _{y \in d d_{v_{i}}^{v}} \sqrt{1-R_{H}^{2}(x, y)}\right\}$ $=\inf \left\{\underline{R_{H}^{(t)}} d_{v_{i}}(x), \inf _{y \in d v_{v_{i}}^{v}} \sqrt{\left.1-R_{H}^{2}(x, y)\right\}}\right.$.

According to Proposition 2,

$\overline{R_{H}^{(t+1)}} d_{v_{i}}^{\prime}(x)=\sup _{y \in d_{v_{i}}^{\prime}} R_{H}(x, y)$.

$\overline{R_{H}^{(t)}} d_{v_{i}}(x)=\sup _{y \in d_{v_{i}}} R_{H}(x, y)=\sup _{y \in d_{v_{i}}^{\prime} \text { or } y \in d_{v_{i}}^{v}} R_{H}(x, y)=\sup \left\{\sup _{y \in d_{v_{i}}^{\prime}} R_{H}(x, y), \sup _{y \in d_{v_{i}}^{v}} R_{H}(x, y)\right\}=\sup \left\{\overline{R_{H}^{(t+1)}} d_{v_{i}}^{\prime}(x), \sup _{y \in d_{v_{i}}^{v}} R_{H}(x, y)\right\}$.

It is clear that there are the following two cases:

- $\overline{R_{H}^{(t)}} R_{H}(x, y)=\overline{R_{H}^{(t+1)}} R_{H}(x, y)>\sup _{y \in d_{v_{i}^{v}}^{v}} R_{H}(x, y)$, then $\overline{R_{H}^{(t+1)}} R_{H}(x, y)=\overline{R_{H}^{(t)}} R_{H}(x, y)$.

- $\overline{R_{H}^{(t)}} R_{H}(x, y)=\sup _{y \in d d_{v_{i}}^{\vee}} R_{H}(x, y) \geq \overline{R_{H}^{(t+1)}} R_{H}(x, y)$, then $\overline{R_{H}^{(t+1)}} R_{H}(x, y)=\sup _{y \in d_{v_{i}}^{\prime}} R_{H}(x, y)$.

(2) $\forall d_{v_{i}}^{\vee} \in\left\{d_{v_{1}}^{\vee}, \ldots, d_{v_{q}}^{\vee}\right\}$, let $d_{v_{i}}$ be refined to $\left\{d_{v_{i}}^{\prime}, d_{v_{i}}^{\vee}\right\}$ at time $t+1$, where $d_{v_{i}}=d_{v_{i}}^{\prime} \cup d_{v_{i}}^{\vee}$.

Because $d_{v_{i}}=d_{v_{i}}^{\prime} \cup d_{v_{i}}^{\vee}, \overline{v_{v_{i}}}=\overline{d_{v_{i}}^{\prime}} \cap \overline{d_{v_{i}}^{\vee}}$ and $\overline{d_{v_{i}}^{\vee}}=\overline{d_{v_{i}}^{\vee}} \cap \overline{d_{v_{i}}^{\prime}} \cup{\overline{v_{i}}}_{1}^{\prime}=\overline{d_{v_{i}}} \cup d_{v_{i}}^{\prime}$. According to Proposition 2,

$\underline{R_{H}^{(t+1)}} d_{v_{i}}^{\vee}(x)=\inf _{y \notin d v_{v_{i}}^{v}} \sqrt{1-R_{H}^{2}(x, y)}=\inf _{y \notin d_{v_{i}} \text { or } y \in d_{v_{v_{i}}}^{\prime}} \sqrt{1-R_{H}^{2}(x, y)}=\inf \left\{\inf _{y \notin d_{v_{i}}} \sqrt{1-R_{H}^{2}(x, y)}, \inf _{y \in d_{v_{i}}^{\prime}} \sqrt{\left.1-R_{H}^{2}(x, y)\right\}}\right.$ $=\inf \left\{\underline{R_{H}^{(t)}} d_{v_{i}}(x), \inf _{y \in d_{v_{i}}^{\prime}} \sqrt{1-R_{H}^{2}(x, y)}\right\}$.

Because $d_{v_{i}}^{\vee}$ is a new decision class, according to Proposition 2,

$\overline{R_{H}^{(t+1)}} d_{v_{i}}^{\vee}(x)=\sup _{y \in d_{v_{i}}^{\vee}} R_{H}(x, y)$.

With respect to the second changing case, there are some decision classes in which some decision values are coarsened.

Proposition 10. Let $\left\{d_{w_{1}}^{(1)}, \ldots, d_{w_{1}}^{(k)}\right\}, \ldots,\left\{d_{w_{r}}^{(1)}, \ldots, d_{w_{r}}^{(l)}\right\}$ be coarsened to $\left\{d_{w_{1}}^{\wedge}, \ldots, d_{w_{s}}^{\wedge}\right\}$ at time $t+1$, respectively, where $d_{w_{i}}^{\wedge}=$ $d_{w_{i}}^{(1)} \cup \ldots \cup d_{w_{i}}^{(k)}, \forall d_{w_{i}}^{\wedge} \in\left\{d_{w_{1}}^{\wedge}, \ldots, d_{w_{s}}^{\wedge}\right\}$, the following results hold for the update of lower and upper approximations.

$$
\begin{aligned}
& \underline{R_{H}^{(t+1)}} d_{w_{i}}^{\wedge}(x)=\inf _{y \notin d} \sqrt{1-R_{w_{i}}{ }^{2}(x, y)}, \\
& \overline{R_{H}^{(t+1)}} d_{w_{i}}^{\wedge}(x)=\sup \left\{\overline{R_{H}^{(t)}} d_{w_{i}}^{(1)}(x), \ldots, \overline{R_{H}^{(t)}} d_{w_{i}}^{(k)}(x)\right\} .
\end{aligned}
$$


Proof 7. $\forall d_{w_{i}}^{\wedge} \in\left\{d_{w_{1}}^{\wedge}, \ldots, d_{w_{s}}^{\wedge}\right\}$, let $\left\{d_{w_{i}}^{(1)}, \ldots, d_{v_{i}}^{(k)}\right\}$ be coarsened to $d_{w_{i}}^{\wedge}$ at time $t+1$ and $d_{w_{i}}^{\wedge}=d_{w_{i}}^{(1)} \cup \ldots \cup d_{w_{i}}^{(k)}$.

Because $d_{w_{i}}^{\wedge}=d_{w_{i}}^{(1)} \cup \ldots \cup d_{w_{i}}^{(k)}, d_{w_{i}}^{\wedge}=d_{w_{i}}^{(1)} \cap \ldots \cap d_{w_{i}}^{(k)}$. According to Proposition 2,

$$
\begin{aligned}
& \underline{R_{H}^{(t+1)}} d_{w_{i}}^{\wedge}(x)=\inf _{y \notin d} \sqrt{1-R_{w_{i}}(x, y)} . \\
& \overline{R_{H}^{(t+1)}} d_{w_{i}}^{\wedge}(x)=\sup _{y \in d_{w_{i}}^{\wedge}} R_{H}(x, y)=\sup _{y \in d_{w_{i}}^{(1)} \cup \ldots \cup d_{w_{i}}^{(k)}} R_{H}(x, y)=\sup \left\{\sup _{y \in d_{w_{i}}^{(1)}} R_{H}(x, y), \ldots, \sup _{y \in d_{w_{i}}^{(k)}} R_{H}(x, y)\right\}=\sup \left\{\overline{R_{H}^{(t)}} d_{w_{i}}^{(1)}(x), \ldots, \overline{R_{H}^{(t)}} d_{w_{i}}^{(k)}\right.
\end{aligned}
$$

Example 7. As shown in Table 3 and based on Example 1, the decision values "Nephrotuberculosis" and "Nephritis" are coarsened to "Nephritis", and the decision value of $x_{9}$ is refined to "NL". At time $t, U / D=\left\{d_{1}, d_{2}, d_{3}\right\}=$ $\left\{d_{w}^{(1)}, d_{w}^{(2)}, d_{v}\right\}, d_{w}^{(1)}=\left\{x_{1}, x_{2}, x_{3}, x_{8}\right\}, d_{w}^{(2)}=\left\{x_{4}, x_{5}\right\}$, and $d_{v}=\left\{x_{6}, x_{7}, x_{9}\right\}$. At time $t+1, U^{\prime} / D^{\prime}=\left\{d_{w}^{\wedge}, d_{3}, d_{v}^{\vee}\right\}=$ $\left\{d_{w}^{\wedge}, d_{v}^{\prime}, d_{v}^{\vee}\right\}, d_{w}^{\wedge}=\left\{x_{1}, x_{2}, x_{3}, x_{8}, x_{4}, x_{5}\right\}, d_{v}^{\prime}=\left\{x_{6}, x_{7}\right\}$, and $d_{v}^{\vee}=\left\{x_{9}\right\}$.

\begin{tabular}{|c|c|c|c|c|c|c|c|c|}
\hline$U$ & $\begin{array}{c}\text { Urinary } \\
\text { Color }\left(a_{1}\right) \\
\end{array}$ & $\begin{array}{c}\text { Urinary } \\
\text { Tuberculosis }\left(a_{2}\right)\end{array}$ & $\begin{array}{l}\mathbf{P H} \\
\left(a_{3}\right) \\
\end{array}$ & $\begin{array}{c}\text { Urinary } \\
\text { Irritation }\left(a_{4}\right) \\
\end{array}$ & $\begin{array}{c}\mathbf{R B C} \\
\left(a_{5}\right) \\
\end{array}$ & $\begin{array}{c}\text { WBC } \\
\left(a_{6}\right) \\
\end{array}$ & $\begin{array}{l}\mathrm{CT} \\
\left(a_{7}\right)\end{array}$ & $d$ \\
\hline$x_{1}$ & Pink & - & 4.0 & $\{\mathrm{M}, \mathrm{U}, \mathrm{P}\}$ & {$[1,4]$} & {$[3,7]$} & & Nephritis \\
\hline$x_{2}$ & Pink & - & 4.5 & $\{\mathrm{M}, \mathrm{U}, \mathrm{P}\}$ & {$[2,4]$} & {$[2,6]$} & & Nephritis \\
\hline$x_{3}$ & Red & $?$ & 3.8 & $\{\mathrm{M}\}$ & {$[1,3]$} & {$[1,4]$} & & Nephritis \\
\hline$x_{4}$ & Pink & + & 3.6 & $\{\mathrm{U}\}$ & {$[2,5]$} & {$[5,7]$} & & \\
\hline$x_{5}$ & Red & + & 3.5 & $\{\mathrm{U}\}$ & {$[1,4]$} & {$[6,7]$} & & \\
\hline$x_{6}$ & Yellow & - & 5.5 & $\{\mathrm{U}, \mathrm{P}\}$ & {$[0,2]$} & {$[0,5]$} & & Health \\
\hline$x_{7}$ & Yellow & $?$ & $?$ & $\{\mathrm{P}\}$ & {$[0,2]$} & {$[1,4]$} & & Health \\
\hline$x_{8}$ & Yellow & - & 4.1 & $\{\mathrm{M}, \mathrm{U}, \mathrm{P}\}$ & {$[2,4]$} & {$[4,6]$} & & Nephritis \\
\hline$x_{9}$ & $?$ & - & 5.6 & $\{\mathrm{U}\}$ & {$[0,2]$} & {$[2,4]$} & & $\begin{array}{c}\text { NL } \\
\text { (Health) }\end{array}$ \\
\hline
\end{tabular}

Table 3: The variation of decision attribute values in HIS

According to Proposition 10,

$\frac{R_{H}^{(t+1)}}{\wedge} d_{w}^{\wedge}\left(x_{1}\right)=\inf _{y \notin d_{w}^{\wedge}} \sqrt{1-R_{H}^{2}\left(x_{1}, y\right)}=0.96$.
$\overline{R_{H}^{(t+1)}} d_{w}^{\wedge}\left(x_{1}\right)=\sup \left\{\overline{R_{H}^{(t)}} d_{w}^{(1)}\left(x_{1}\right), \overline{R_{H}^{(t)}} d_{w}^{(2)}\left(x_{1}\right)\right\}=\sup \left\{\overline{R_{H}^{(t)}} d_{1}\left(x_{1}\right), \overline{R_{H}^{(t)}} d_{2}\left(x_{1}\right)\right\}=\sup \{1,0.31\}=1$.

According to Proposition 9,

$\underline{R_{H}^{(t+1)} d_{v}^{\prime}}\left(x_{6}\right)=\inf \left\{\underline{R_{H}^{(t)}} d_{3}\left(x_{6}\right), \inf _{y \in d_{v}^{\vee}} \sqrt{1-R_{H}^{2}\left(x_{6}, y\right)}=\left\{0.95, \sqrt{1-R_{H}^{2}\left(x_{6}, x_{9}\right)}\right\}=0.87\right.$.

$\overline{R_{H}^{(t+1)}} d_{v}^{\prime}\left(x_{6}\right)=\sup _{y \in d_{v}^{\prime}} R_{H}^{2}\left(x_{6}, y\right)=\sup _{y \in d_{3}-\left\{x_{9}\right\}} R_{H}\left(x_{6}, y\right)$.

$\overline{R_{H}^{(t)}} d_{3}\left(x_{6}\right)=1>R_{H}\left(x_{6}, x_{9}\right)=0.49$. Therefore, $\overline{R_{H}^{(t+1)}} d_{v}^{\prime}\left(x_{6}\right)=1$.

$\underline{R_{H}^{(t+1)} d_{v}^{\vee}}\left(x_{1}\right)=\inf \left\{\underline{R_{H}^{(t)}} d_{3}\left(x_{1}\right), \sup _{y \in d_{v}^{\prime}} \sqrt{1-R_{H}^{2}(x, y)}\right\}=\inf \left\{0, \sup _{y \in d_{v}^{\prime}} \sqrt{1-R_{H}^{2}(x, y)}\right\}=0$. 


$$
\overline{R_{H}^{(t+1)}} d_{v}^{\vee}\left(x_{1}\right)=\sup _{y \in d_{v_{i}}^{\vee}} R_{H}\left(x_{1}, x_{9}\right)=0.21
$$

Similarly, the approximations at time $t+1$ are as follows:

$$
\begin{aligned}
& R_{H}^{(t+1)} d_{w}^{\wedge}=\left\{0.96 / x_{1}, 0.97 / x_{2}, 0.98 / x_{3}, 0.99 / x_{4}, 0.95 / x_{5}, 0 / x_{6}, 0 / x_{7}, 0.90 / x_{8}, 0 / x_{9}\right\} . \\
& \bar{R}_{H}^{(t+1)} d_{v}^{\prime}=\left\{0 / x_{1}, 0 / x_{2}, 0 / x_{3}, 0 / x_{4}, 0 / x_{5}, 0.87 / x_{6}, 0.99 / x_{7}, 0 / x_{8}, 0 / x_{9}\right\} . \\
& \overline{R_{H}^{(t+1)}} d_{v}^{\vee}=\left\{0 / x_{1}, 0 / x_{2}, 0 / x_{3}, 0 / x_{4}, 0 / x_{5}, 0 / x_{6}, 0 / x_{7}, 0 / x_{8}, 0.87 / x_{9}\right\} . \\
& \overline{\overline{R_{H}^{(t+1)}}} d_{w}^{\wedge}=\left\{1 / x_{1}, 1 / x_{2}, 1 / x_{3}, 1 / x_{4}, 1 / x_{5}, 0.31 / x_{6}, 0.10 / x_{7}, 1 / x_{8}, 0.21 / x_{9}\right\} . \\
& \frac{R_{H}^{(t+1)}}{R_{v}^{\prime}}=\left\{0.28 / x_{1}, 0.23 / x_{2}, 0.18 / x_{3}, 0.05 / x_{4}, 0.08 / x_{5}, 1 / x_{6}, 1 / x_{7}, 0.31 / x_{8}, 0.49 / x_{9}\right\} . \\
& R_{H}^{(t+1)} d_{v}^{\vee}=\left\{0.21 / x_{1}, 0.18 / x_{2}, 0.14 / x_{3}, 0.06 / x_{4}, 0.08 / x_{5}, 0.49 / x_{6}, 0.11 / x_{7}, 0.10 / x_{8}, 1 / x_{9}\right\} .
\end{aligned}
$$

When decision attribute values are refined or coarsened, the algorithm does not generate the fuzzy relations again. According to the above propositions, the algorithm for Incremental Updating Approximations based on FRS under the Variation of Decision Attribute Values (IUAFRS-VDAV) is outlined in Algorithm 4.

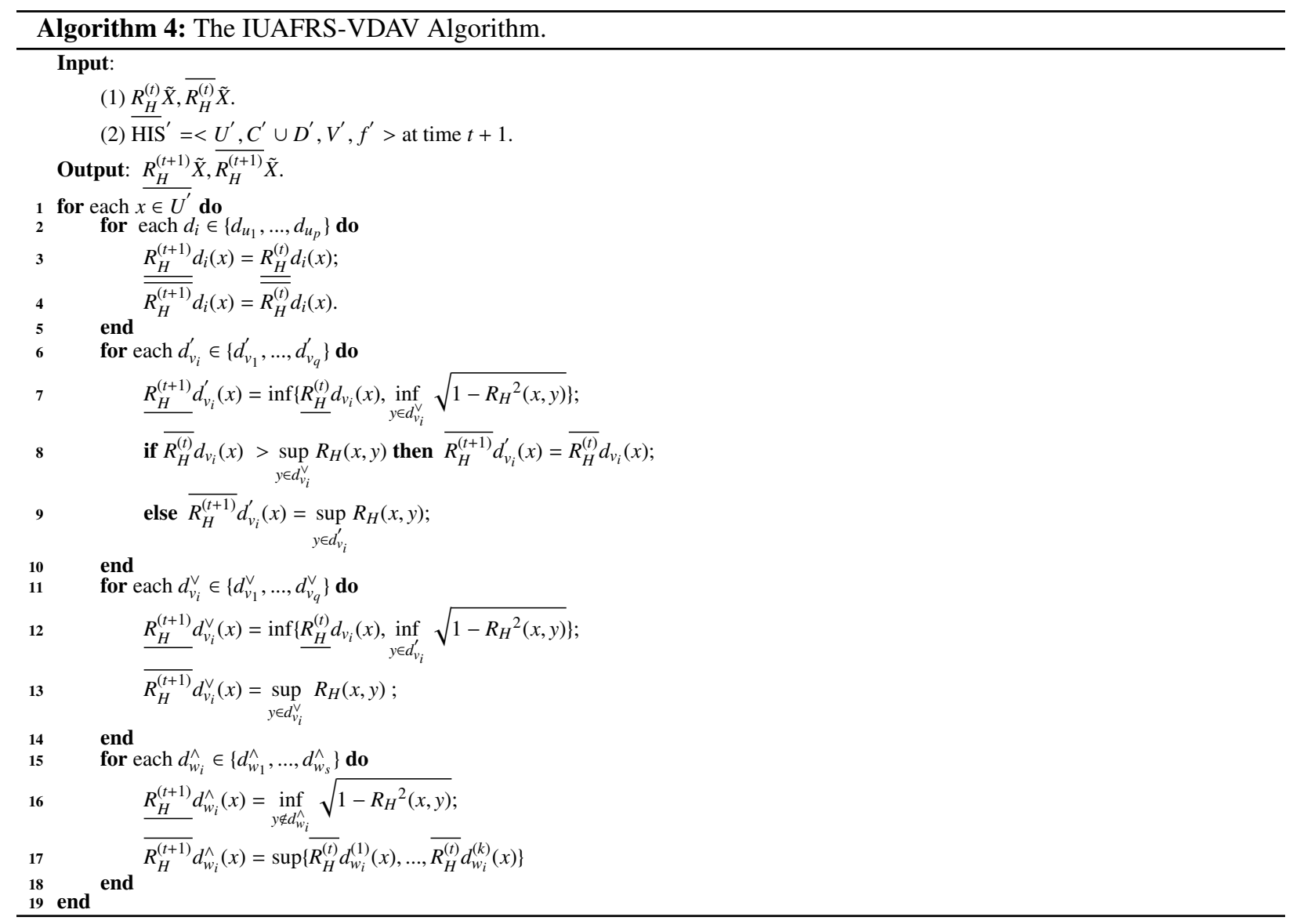

According to Proposition 8, Lines 2-5 compute the lower and upper approximations of the decision classes $d_{i} \in$ $\left\{d_{u_{1}}, \ldots, d_{u_{p}}\right\}$. It is clear that the time complexity is $O(n)$. Let decision classes $d_{v_{1}}, \ldots, d_{v_{q}}$ be refined to $\left\{d_{v_{1}}^{\prime}, d_{v_{1}}^{\vee}\right\}, \ldots$, $\left\{d_{v_{q}}^{\prime}, d_{v_{q}}^{\vee}\right\}$ at time $t+1$, respectively, where $d_{v_{i}}=d_{v_{i}}^{\prime} \cup d_{v_{i}}^{\vee}$. According to Proposition 9, Lines 6-10 compute the lower and upper approximations of the decision classes $d_{v_{i}}^{\prime} \in\left\{d_{v_{1}}^{\prime}, \ldots, d_{v_{q}}^{\prime}\right\}$. The time complexity is $\mathrm{O}\left(n \times \max \left(\log \left|d_{v_{i}}^{\vee}\right|, \log \left|d_{v_{i}}^{\prime}\right|\right)\right) \approx$ $O(n \times \log n)$. Lines 11-14 compute the lower and upper approximations of the decision classes $d_{v_{i}}^{\vee} \in\left\{d_{v_{1}}^{\vee}, \ldots, d_{v_{q}}^{\vee}\right\}$. The time complexity is $O(n \times \log n)$. According to Proposition 10, Lines 15-18 compute the lower and upper approximations of the decision classes $d_{w_{i}}^{\wedge} \in\left\{d_{w_{1}}^{\wedge}, \ldots, d_{w_{s}}^{\wedge}\right\}$. The time complexity is $O\left(n \times \log \mid d_{w_{i}}^{\wedge}\right)$. Therefore, the time complexity of Algorithm 4 is $O(n \times \log n)$. It is clear that the incremental algorithm is superior to the non-incremental algorithm. 


\section{Experimental evaluation}

In this section, we compare the computational time of the non-incremental algorithm (NIUAFRS) and the proposed incremental algorithms (IUAFRS-VCAV and IUAFRS-VDAV) on different data sets. Moreover, we compare the computational time and the approximation classification accuracies of IUAFRS-VCAV and an incremental algorithm called Rough Set based Dynamic Maintenance Approach for Approximations in Coarsening and Refining Attribute Values (RSDMAA-CRAV).

Experiments were performed on a $2.2 \mathrm{GHz}$, eight core and 16 thread server with $8 \mathrm{~GB}$ of memory, running Windows 7. Algorithms were coded in SQL Server 2008. The eight data sets (including hybrid features) used herein came from the University of California, Irvine (UCI) Machine Learning Repository (http://www.ics.uci.edu/); see Table 4. To evaluate the capability of dealing with the set-valued attributes, we composed new data sets Credit (set) based on Credit. In Credit, three Boolean attributes $(1,9,10)$ are combined into a new set-valued attribute 16 (shown as Credit (set) in Table 4). The experiments were conducted in a 10-fold cross validation mode. When computing the fuzzy relations with the Gaussian kernel, the kernel parameter $\delta$ must be specified. In [16], Hu et al. gave an approach for setting $\delta$. Therefore, according to their approach, we consider random values of $\delta$ from $[0.1,0.36]$.

Table 4: Descriptions of data sets.

\begin{tabular}{clcclcc}
\hline ID & Data set & Samples & Features & Feature Types & Decision Classes & Divided Classes \\
\hline 1 & Wine & 178 & 18 & Boolean, Real & 3 & 10 \\
2 & Heparits & 155 & 19 & Categorical, Boolean, Real & 2 & 10 \\
3 & Horse & 368 & 26 & Categorical, Boolean, Real & 2 & 10 \\
4 & Iono & 351 & 34 & Boolean, Real & 2 & 10 \\
5 & Hill_Valley & 606 & 100 & Real & 2 & 10 \\
6 & Credit (set) & 690 & 16 & Categorical, Boolean, Real, Set & 2 & 10 \\
7 & German & 1000 & 20 & Categorical, Boolean, Real & 2 & 10 \\
8 & Sick & 2800 & 28 & Categorical, Boolean, Real & 2 & 10 \\
9 & Bands & 540 & 40 & Categorical, Boolean, Real & 2 & 10 \\
\hline
\end{tabular}

\subsection{A comparison of NIUAFRS and IUAFRS-VCAV under the variation of condition attribute values}

To analyze the efficiency of NIUAFRS and IUAFRS-VCAV, we ran the two algorithms on the nine data sets in Table 4 and let select condition attribute values vary in each of the nine data sets. The experimental results are presented in Figure 1, where $N_{c v}$ denotes the number of objects whose condition attribute values vary and $N$ denotes the total number of the samples in the data set. The variation ratio is $R_{c v}=N_{c v} / N$.

In each subfigure of Figure 1, the $x$-coordinate is the variation ratio $R_{c v}$, while the $y$-coordinate is the elapsed time. Figure 1 displays the more detailed change trends of each of the two algorithms for increasing values of $R_{c v}$.

As shown in Figure 1, the elapsed time of IUAFRS-VCAV is smaller than that of NIUAFRS. The elapsed time of IUAFRS-VCAV increases with increasing values of $R_{c v}$. As $R_{c v}$ increases, the elapsed time of IUAFRS-VCAV approaches that of NIUAFRS.

\subsection{Comparisons of IUAFRS-VCAV and RSDMAA-CRAV under the variation of condition attribute values}

In recent years, based on Pawlak's RST or DRSA, some incremental algorithms for updating approximations have been presented under the variation of attribute values $[8,21,32,35]$. The incremental algorithms based on DRSA are designed for knowledge discovery of ordered information, but our algorithm is not designed in this manner. Therefore, it is difficult to compare these algorithms with ours. Based on Pawlak's RST, Chen et al. proposed RSDMAA-CRAV 
[8]. To analyze the elapsed time and approximation classification accuracies of RSDMAA-CRAV and IUAFRSVCAV, we ran the two algorithms on the nine data sets in Table 4. In RSDMAA-CRAV, each real value and set value is converted into a categorical value. As shown in Figure 1, as $R_{c v}$ increases, the elapsed time of IUAFRS-VCAV and RSDMAA-CRAV become closer.
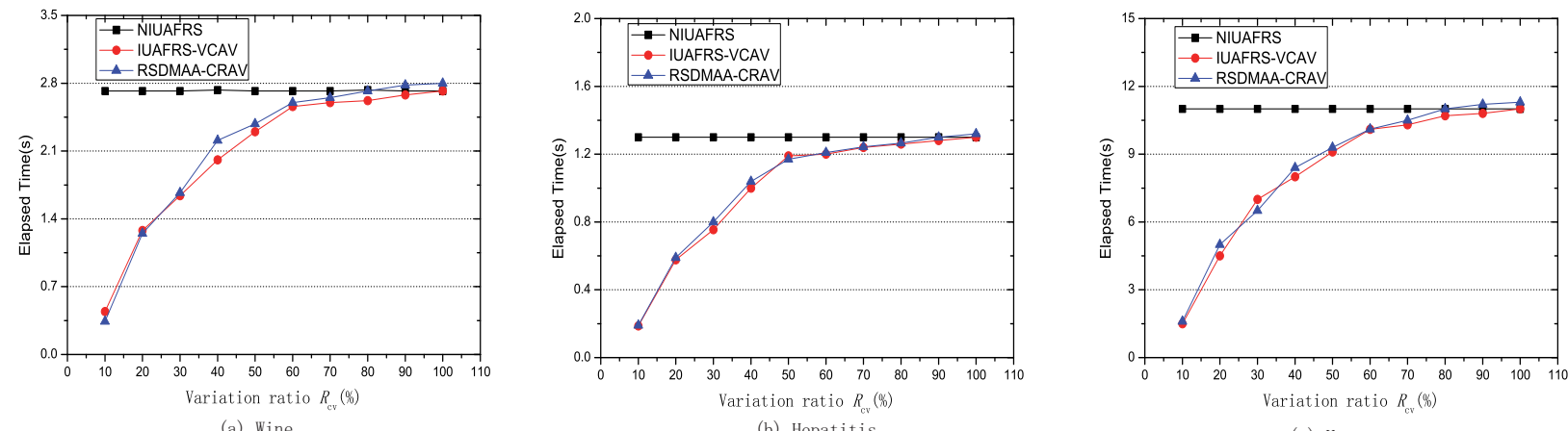

(b) Hepatitis
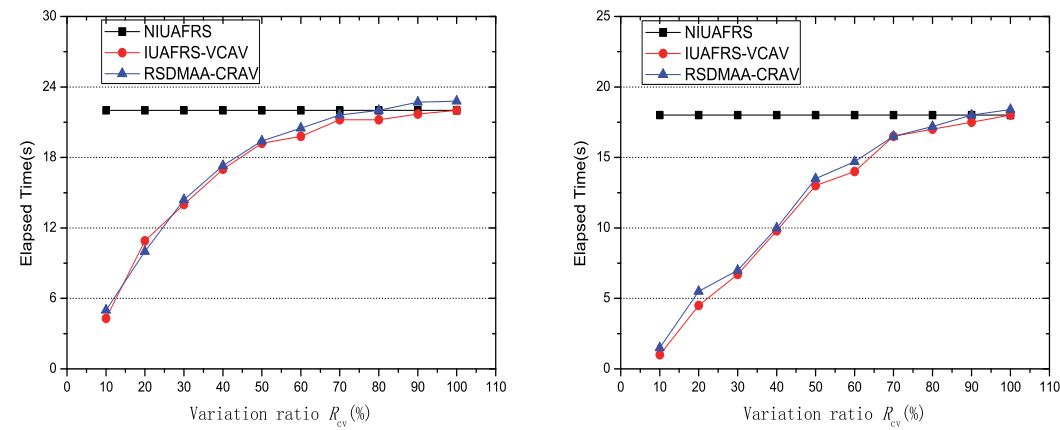

(c) Horse

(d) Iono
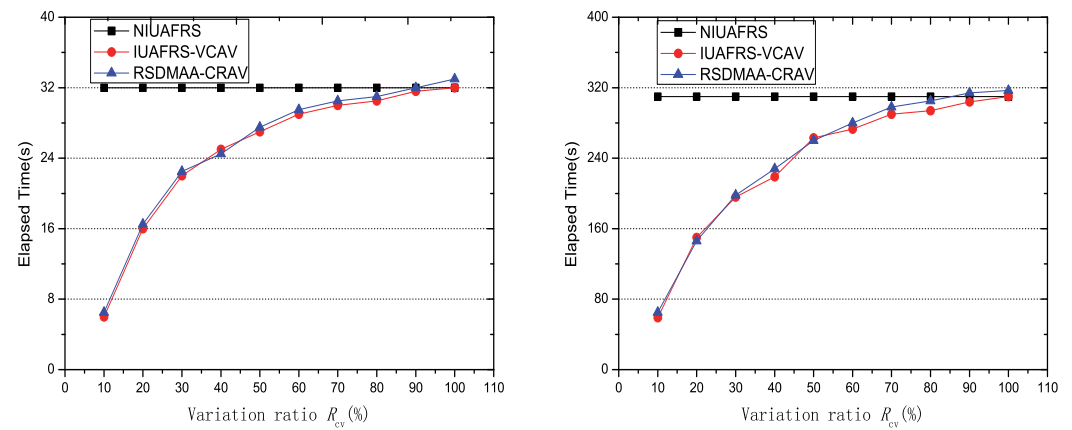

(g) German

(h) Sick

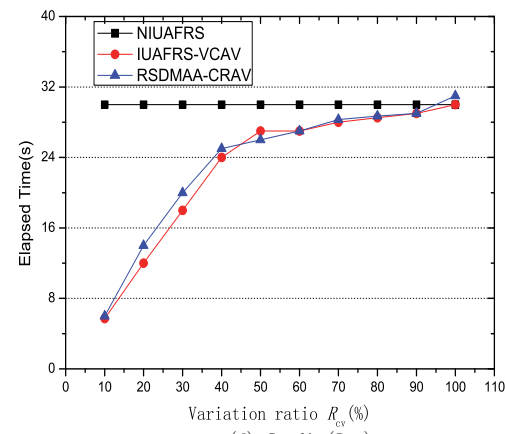

(f) $\operatorname{Credit}(\mathrm{Set})$

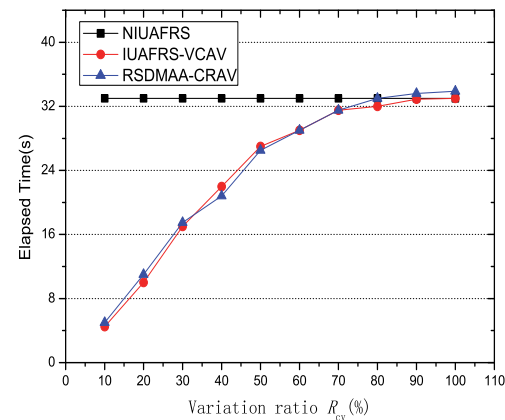

(i) Bands

Figure 1: Average elapsed time of NIUAFRS, IUAFRS-VCAV and RSDMAA-CRAV versus the variation of condition attribute values.

In Figure 2, the $x$-coordinate is the data set, while the $y$-coordinate is the accuracies of approximation classification. Notice that the approximation classification accuracies of IUAFRS-VCAV outperform RSDMAA-CRAV in most data sets. 


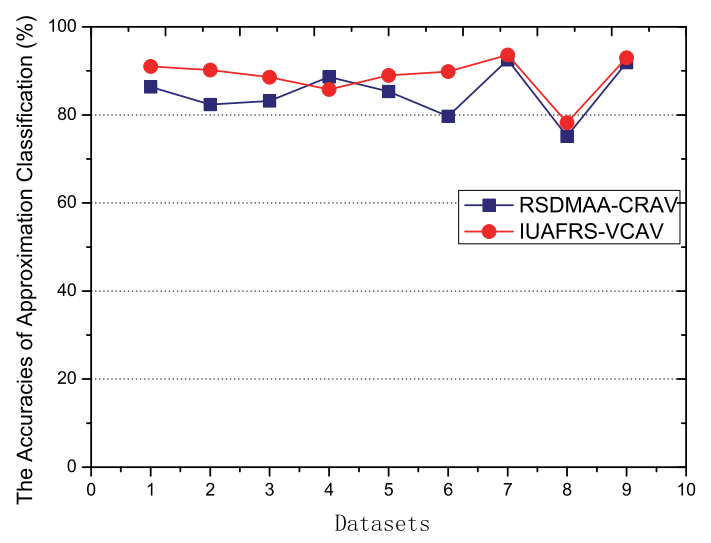

Figure 2: Comparisons of RSDMAA-CRAV and IUAFRS-VCAV versus the variation of condition attribute values

\subsection{A comparison of NIUAFRS and IUAFRS-VDAV under the variation of decision values}

To analyze the efficiency of NIUAFRS and IUAFRS-VDAV, we ran the two algorithms on the nine data sets in Table 4 , and the decision classes of each data set were divided randomly into 10 classes. $R_{i}$ denotes the number of refining decision classes, where $i=1,2, \ldots, 10$ and $C_{i}$ denotes the number of coarsening decision classes, where $i=2, \ldots, 9$. The experimental results of refining and coarsening decision values are presented in Figures 3 and 4 , respectively.

In each subfigure of Figure 3, the $x$-coordinate is the refining number $R_{i}$, while the $y$-coordinate is the elapsed time. Figure 3 displays the more detailed change trends of each of the two algorithms for increasing values of $R_{i}$. As shown in Figure 3, for increasing values of $R_{i}$, the elapsed times of IUAFRS-VDAV and NIUAFRS increase slightly. The elapsed time of IUAFRS-VCAV is always significantly smaller than NIUAFRS. Clearly, the proposed incremental algorithm significantly outperforms the non-incremental algorithm.

In each subfigure of Figure 4, the $x$-coordinate is the coarsening number $C_{i}$, while the $y$-coordinate is the elapsed time. Figure 4 displays the more detailed change trends of each of the two algorithms for increasing values of $C_{i}$. As shown in Figure 4, for increasing values of $C_{i}$, the elapsed time of IUAFRS-VDAV increases slightly, but that of NIUAFRS decreases slightly. When decision values are coarsened, in general, the incremental algorithm IUAFRSVDAV significantly outperforms the non-incremental algorithm. 

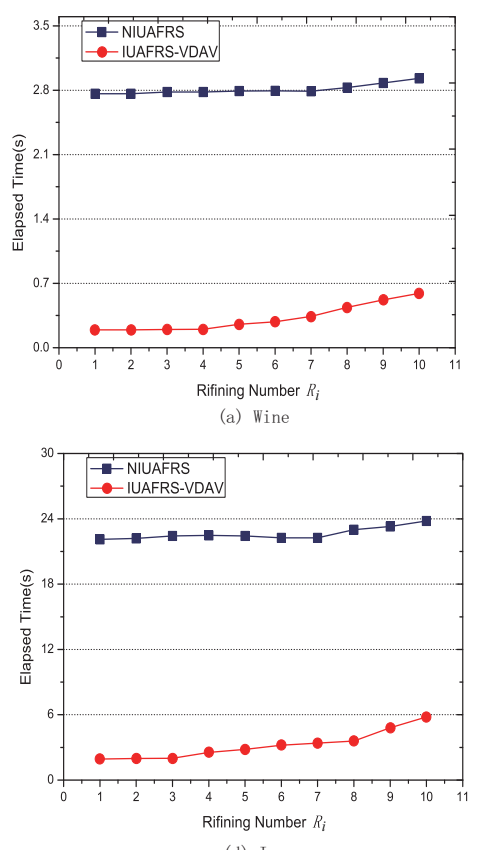

(d) Iono

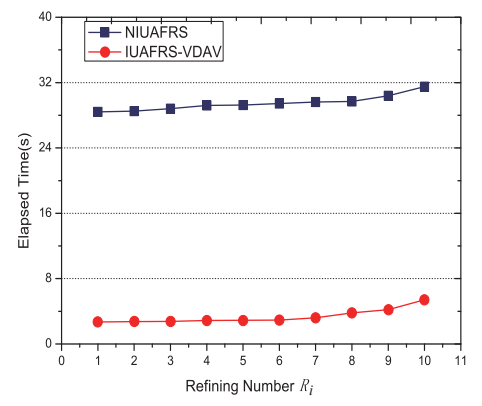

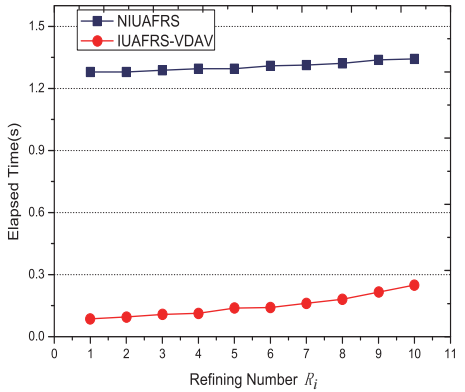

(b) Hepatitis

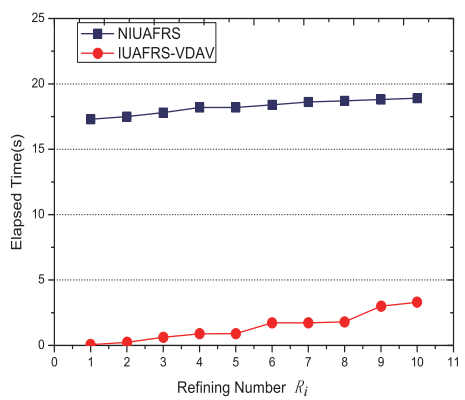

(e) Hill Valley

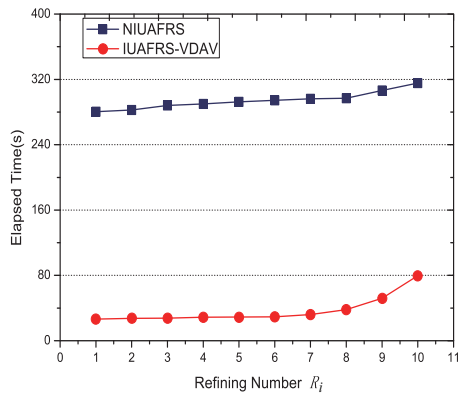

(h) Sick
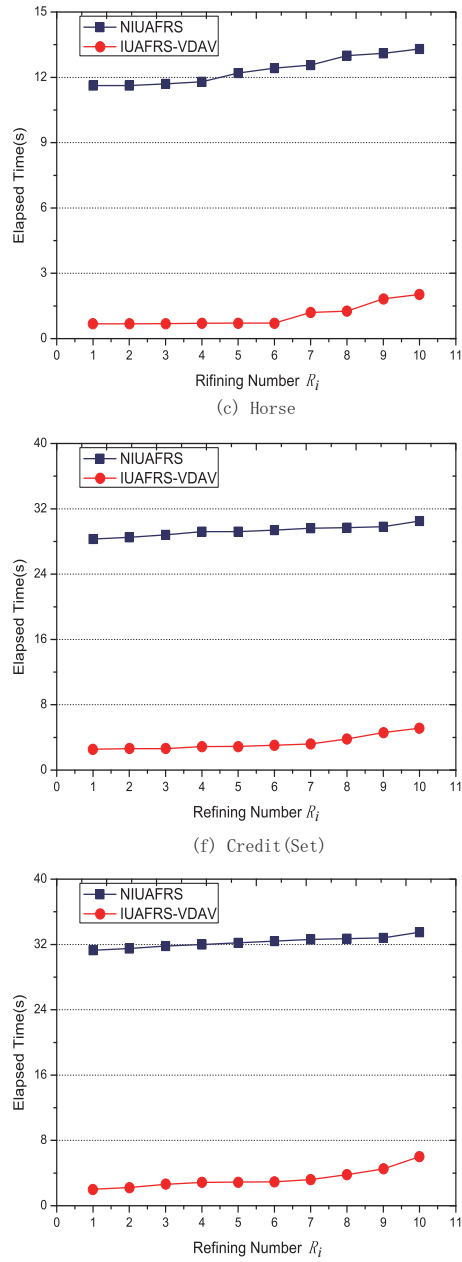

Figure 3: Average elapsed time of NIUAFRS and IUAFRS-VDAV versus the refining of decision values 

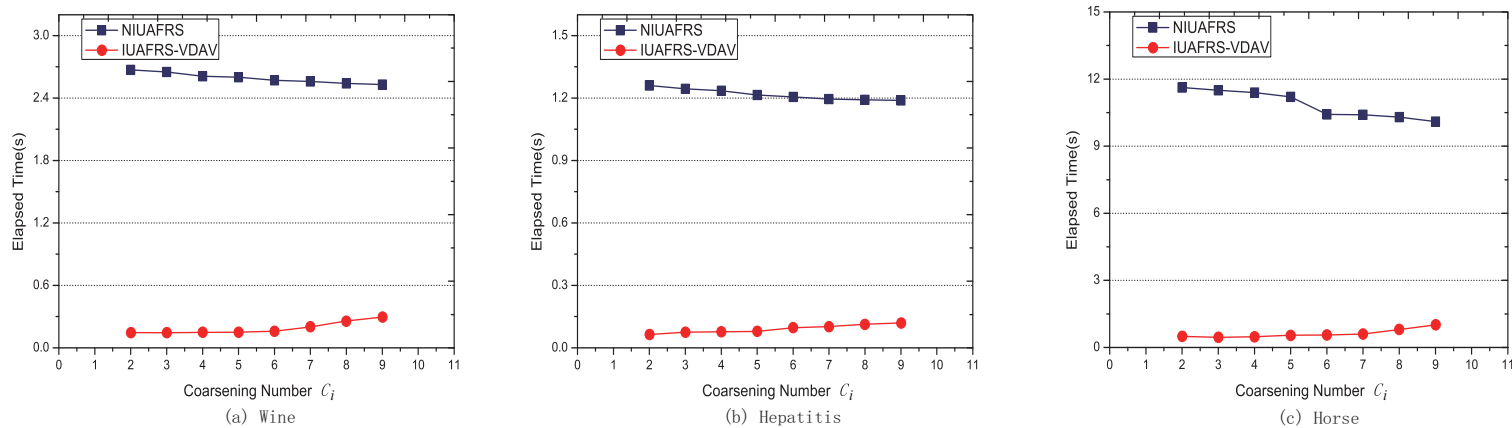

(b) Hepatitis
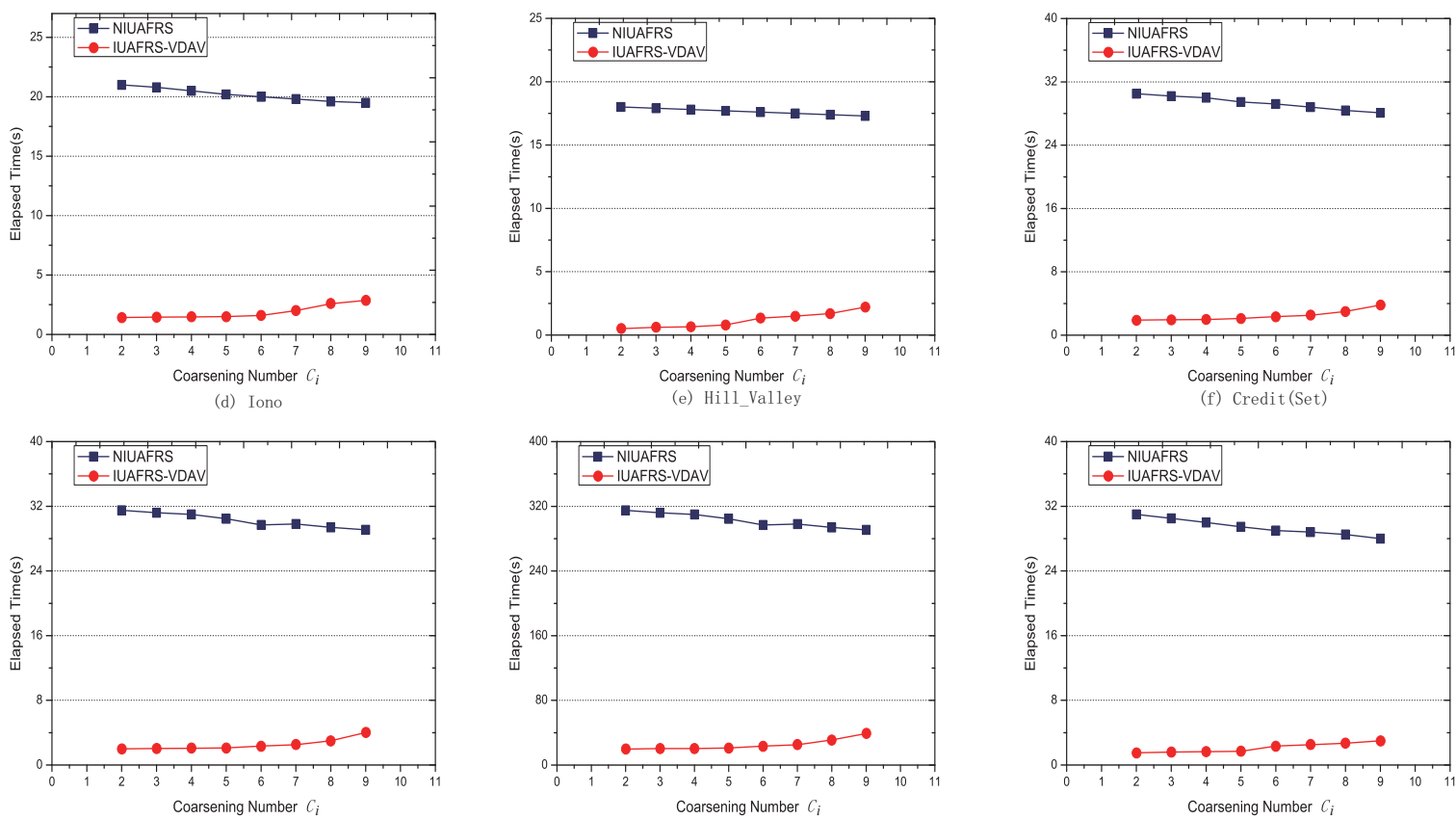

(g) German

(h) Sick

(i) Bands

Figure 4: Average elapsed time of NIUAFRS and IUAFRS-VDAV versus the coarsening of decision values

\section{Conclusions}

By considering a decision system characterized by both hybrid attributes and missing values, a new HD-I distance was constructed to handle this particular type of HIS. Combined with the concepts of HD-I and the Gaussian kernel, a novel FRS model was proposed. Meanwhile, the incremental updating mechanism of fuzzy rough approximations was investigated with respect to the variation of attribute values in an HIS. Several examples were further employed to illustrate the validity of the proposed incremental methods. Then, the corresponding incremental algorithms (IUAFRS-VCAV and IUAFRS-VDAV) were proposed. Experimental studies on eight UCI data sets and one artificial data set showed that the incremental algorithms can improve the computational efficiency for updating approximations when some attribute values in HIS vary over time. We validated that the proposed approaches can be applied effectively for knowledge discovery in the dynamic HIS. In the future, parallel computing technologies, e.g., MapReduce [45, 50], for the optimization of the incremental updating approach, under the variation of domain of attributes or objects in HIS will be taken into consideration.

\section{Acknowledgement}

This work is supported by the National Science Foundation of China (Nos. 61573292, 61572406, 71571148), the Scientific Research Foundation of Sichuan Provincial Education Department (Nos. 16ZA0328, 16ZA0329), and the 
Scientific Research Foundation of Yibin University (Nos. 2015YY01, 2015QD08).

\section{References}

[1] L. An, L. Tong, W. Fan, Mining decision rules based on in-sim-dominance relations, in: Progress in Intelligence Computation and Applications, Wuhan, China, 2007, pp. 448-452.

[2] M. Bernhard, On the T-transitivity of kernels, Fuzzy Sets and Systems 157 (2006) 787-796.

[3] C.C. Chan, A rough set approach to attribute generalization in data mining, Information Sciences 107 (1998) $177-194$

[4] S.L. Chen, Fuzzy theory and its applications, Beijing: Science Press, 2006.

[5] H.M. Chen, T.R. Li, J.B. Z. C. Luo, X.J. Li, Probabilistic composite rough set and attribute reduction, in: Proceedings of Seventh International Conference on Intelligent Systems and Knowledge Engineering, Beijing, China, 2012, pp. 189-197.

[6] H.M. Chen, T.R. Li, C. Luo, S.J. Horng, G.Y. Wang, A decision-theoretic rough set approach for dynamic data mining, IEEE Transactions on Fuzzy Systems, 23 (6) (2015) 1958-1970.

[7] H.M. Chen, T.R. Li, C.X. Hu, X.L. Ji, An incremental updating principle for computing approximations in information systems while the object set varies with time, in: Proceedings of 2009 IEEE International Conference on Granular Computing, Nanchang, China, 2009, pp. 49-52.

[8] H.M. Chen, T.R. Li, S.J. Qiao, D. Ruan, A rough set based dynamic maintenance approach for approximations in coarsening and refining attribute values, International Journal of Intelligent Sytems 25 (2010) 1005-1026.

[9] Y. Cheng, The incremental method for fast computing the rough fuzzy approximations, Data and Knowledge Engineering 70 (2011) 84-100.

[10] J.H. Dai, W.T. Wang, X. Qing, H.W. Tian, Uncertainty measurement for interval-valued decision systems based on extended conditional entropy, Knowledge-Based Systems 27 (2012) 443-450.

[11] T.R. Li, C. Luo, H.M. Chen, J.B. Zhang, PICKT: A solution for big data analysis, Lecture Notes in Computer Science 9436 (2015) 15-25.

[12] D. Dubois, H. Prade, Rough fuzzy sets and fuzzy rough sets, International Journal of General Systems 17 (2-3) (1990) 191-209.

[13] S. Greco, B. Matarazzo, R. Slowinski, Rough approximation of a preference relation by dominance relations, European Journal of Operational Research 117 (1) (1999) 63-83.

[14] Q.H. Hu, D.R. Yu, J.F. Liu, C.X. Wu, Neighborhood rough set based heterogeneous feature subset selection, Information Sciences 178 (18) (2008) 3577-3594.

[15] Q.H. Hu, D.R. Yu, W. Pedrycz, D.G. Chen, Kernelized fuzzy rough sets and their applications, IEEE Transactions on Knowledge and Data Engineering 23 (11) (2011) 1649-1667.

[16] Q.H. Hu, L. Zhang, D.G. Chen, W. Pedrycz, D.R. Yu, Gaussian kernel based fuzzy rough Sets: Model, uncertainty measures and applications, International Journal of Approximate Reasoning 51 (2010) 453-471.

[17] Q.H. Hu, Z.X. Xie, D.R. Yu, Hybrid attribute reduction based on a novel fuzzy-rough model and information granulation, Pattern recognition 40 (12) 2007 (3509-3521).

[18] X. Hu, W. Pedrycz, O. Castillo, P. Melin, Fuzzy rule-based models with interactive rules and their granular generalization, Fuzzy Sets and Systems, DOI: 10.1016/j.fss.2016.03.005, 2016

[19] C.R. Li, G.D. Duan, F.J. Zhong, Rotation invariant texture retrieval considering the scale dependence of gabor wavelet, IEEE Transactions on Image Processing 24 (8) (2015) 2344-2354.

[20] S.Y. Li, T.R. Li, D. Liu, Dynamic maintenance of approximations in dominance-based rough set approach under the variation of the object set, International Journal of Intelligent Systems 28 (8) (2013) 729-751.

[21] S.Y. Li, T.R. Li, D. Liu, Incremental update of approximations in dominance-based rough sets approach under the variation of attribute values, Information Sciences 294 (2015) 348-361.

[22] S.Y. Li, T.R. Li, D. Liu, Incremental updating approximations in dominance-based rough sets approach under the variation of the attribute set, Knowledge-Based Systems 40 (2013) 17-26.

[23] S.Y. Li, T.R. Li, J. Hu, Update of approximations in composite information systems, Knowledge-Based Systems 83 (2015) $138-148$.

[24] S.Y. Li, T.R. Li, Z.X. Zhang, H.M. Chen, J.B. Zhang, Parallel computing of approximations in dominance-based rough sets approach, Knowledge-based Systems 87 (2015) 102-111.

[25] T.R. Li, D. Ruan, G. Wets, J. Song, Y. Xu, A rough sets based characteristic relation approach for dynamic attribute generalization in data mining, Knowledge-Based Systems 20 (5) (2007) 485-494.

[26] T.R. Li, Y. Xu, A generalized rough set approach to attribute generalization in data mining, Journal of Southwest Jiaotong University 8 (1) (2000) 69-75.

[27] X.N. Li, H.J. Yi, S.Y. Liu, Rough sets and matroids from a lattice-theoretic view point, Information Sciences $342(2016) 37-52$.

[28] T.Y. Lin, Q. Liu, K.J. Huang, Rough sets, neighborhood systems and approximation, in: Proceedings of the Fifth International Symposium on Methodologies of Intelligent Systems, 1990, pp. 130-141.

[29] D. Liu, T.R. Li, D. Ruan, J.B. Zhang, Incremental learning optimization on knowledge discovery in dynamic business intelligent systems, Journal of Global Optimization 51 (2011) 325-344.

[30] D. Liu, T.R. Li, G.R. Liu, P. Hu, An approach for inducing interesting incremental knowledge based on the change of attribute values, in: Proceedings of 2009 IEEE International Conference on Granular Computing (GRC2009), Nanchang, China, 2009, pp. 415-418.

[31] D. Liu, T.R. Li, H.M. Chen, X.L. Ji, Approaches for incremental learning knowledge on the changing attribute values, in: Proceedings of the 4th Intelligent Systems and Knowledge Engineering (ISKE2009), Hasselt, Belgium, 2009, pp. 94-99.

[32] C. Luo, L.X. Lu, T.R. Li, A.P. Zeng, H.M. Chen, An approach for incremental maintenance of approximations in set-valued ordered decision systems while updating criteria values, in: Proceedings of the 2013 Joint IFSA World Congress and NAFIPS Annual Meeting (IFSA/NAFIPS), Edmonton, Alberta, Canada, 2013, pp. 819-824.

[33] C. Luo, T.R. Li, H.M. Chen, D. Liu, Incremental approaches for updating approximations in set-valued ordered information systems, Knowledge-Based Systems 50 (2013) 218-233. 
[34] C. Luo, T.R. Li, H.M. Chen, Dynamic maintenance of approximations in set-valued ordered decision systems under the attribute generalization, Information Sciences 257 (2014) 210-228.

[35] C. Luo, T.R. Li, H.M. Chen, L.X. Lu, Fast algorithms for computing rough approximations in set-valued decision system while updating criteria values, Information Sciences 299 (2015) 221-242.

[36] Z. Pawlak, Rough sets, International Journal of Computer and Information Science 11 (5) (1982) 341-356.

[37] W. Pedrycz, Granular computing: Analysis and design of intelligent systems, CRC Press/Francis Taylor, Boca Raton, 2013.

[38] W. Pedrycz, R. Al-Hmouz, A.S. Balamash, A. Morfeq, Designing granular fuzzy models: A hierarchical approach to fuzzy modeling, Knowledge-Based Systems 76 (2015), 42-52.

[39] J. Stefanowski, A. Tsoukias, On the extension of rough sets under incomplete information, Lecture Notes in Artificial Intelligence 1711 (1999) 73-81.

[40] B.Z. Sun, Z.T. Gong, D.G. Chen, Fuzzy rough set theory for the interval-valued fuzzy information systems, Information Sciences 178 (2008) 794-815.

[41] L. Wang, Y. Wu, G.Y. Wang, An incremental rule acquisition algorithm based on variable precision rough set model, Journal of Chongqing University of Posts and Telecommunications (Natural Science) 17 (6) (2005) 709-713.

[42] D.R. Wilson, T.R. Martinez, Improved heterogeneous distance functions, Journal of Artificial Intelligence Research 6 (1997) 1-34.

[43] A.P. Zeng, T.R. Li, C. Luo, J.B. Zhang, An incremental approach for updating approximations of rough fuzzy set under the variation of attribute values, in: Proceeding of Computational Intelligence in Dynamic and Uncertain Environments (CIDUE 2013), IEEE Press, Symposium, 2013, pp. 54-60.

[44] A.P. Zeng, T.R. Li, D. Liu, J.B. Zhang, H.M. Chen, A fuzzy rough set approach for incremental feature selection on hybrid information systems, Fuzzy Sets and Systems 258 (2015) 39-60.

[45] J.B. Zhang, J.S. Wong, Y. Pan, T.R. Li, A parallel matrix-based method for computing approximations in incomplete information systems, IEEE Transactions on Knowledge and Data Engineering, 27 (2) (2015) 326-339.

[46] J.B. Zhang, T.R. Li, D. Liu, An approach for incremental updating approximations in variable precision rough sets while attribute generalizing, in: Proceedings of 2010 IEEE International Conference on Intelligent Systems and Knowledge Engineering, Hangzhou, China, 2010, pp. 7781

[47] J.B. Zhang, T.R. Li, D. Ruan, D. Liu, Neighborhood rough sets for dynamic data mining, International Journal of Intelligent Systems 27 (2012) 317-342

[48] J.B. Zhang, T.R. Li, H.M. Chen, Composite rough sets, in: Proceedings of 2012 International Conference on Artificial Intelligence and Computational Intelligence, Chengdu, China, 2012, pp. 150-159.

[49] J.B. Zhang, T.R. Li, H.M. Chen, Composite rough sets for dynamic data mining, Information Sciences 257 (2014) $81-100$.

[50] J.B. Zhang, Y. Zhu, Y. Pan, T.R. Li, Efficient parallel boolean matrix based algorithms for computing composite rough set approximations, Information Sciences, 329 (2016) 287-302.

[51] B.T. Zhao, X.J. Chen, Q.S. Zeng, Incomplete hybrid attributes reduction based on neighborhood granulation and approximation, in: Proceedings of 2009 IEEE International Conference on Mechatronics and Automation (ICMA 2009), Changchun, China, 2009.

[52] Z. Zheng, G.Y. Wang, A rough set and rule tree based incremental knowledge acquisition algorithm, Fundamenta Informaticae 59 (2-3) (2004) 299-313. 